\title{
Capacity Model of Exclusive Right-Turn Lane at Signalized Intersection considering Pedestrian-Vehicle Interaction
}

\author{
Chengcheng Yang, Jiawen Wang $(D$, and Jieshuang Dong \\ Business School, University of Shanghai for Science and Technology, Shanghai 200000, China \\ Correspondence should be addressed to Jiawen Wang; wangjw@usst.edu.cn
}

Received 12 November 2019; Revised 21 May 2020; Accepted 11 July 2020; Published 1 August 2020

Academic Editor: Maria Vittoria Corazza

Copyright (c) 2020 Chengcheng Yang et al. This is an open access article distributed under the Creative Commons Attribution License, which permits unrestricted use, distribution, and reproduction in any medium, provided the original work is properly cited.

\begin{abstract}
In high density urban areas, pedestrians have a great influence on the capacity of intersections. This paper studies the influence of pedestrians on road capacity and proposes an exclusive right-turn lane capacity model considering pedestrian-vehicle interaction (PV-RTC). Firstly, a pedestrian-vehicle interaction (PVI) model is proposed based on the logit model and static games theory of incomplete information. Through this model, the probability of 6 kinds of pedestrian-vehicle interaction situations (vehicles yield to pedestrians, pedestrians yield to vehicles, etc.) in the crosswalk can be obtained. Then, based on the basic idea of the stop line method and the probabilities of above situations, the PV-RTC model is established, and the sensitivity analysis of the important factors (pedestrian arrival rate, yielding rate, and green time ratio) affecting the model is carried out to clarify the mechanism of the proposed model. Finally, a pedestrian-vehicle interaction model of cellular automata for the exclusive right-turn lane is established and its simulation results are compared with the results of the PV-RTC model. The results show that the relative error between the microscopic simulation model and PV-RTC model is less than 15\% overall, which verifies the validity of the PV-RTC model. This study provides references for a more precise estimation method of pedestrian impact on road capacity.
\end{abstract}

\section{Introduction}

With the continuous aggravation of traffic congestion in big cities and the improvement of sustainable development requirements such as rational land use, energy conservation, and environmental protection, more and more attention has been paid on using limited road resources to relieve traffic congestion and improving the operation efficiency of the traffic system. Intersections play a significant role in the operation of road networks [1]. The traffic flow efficiency at these spots largely determines the traffic efficiency of the whole road network; they are the main bottlenecks in the system [1]. Correctly analyzing and calculating the capacity of intersections and rebuilding or building new intersections accordingly are of great significance for improving the efficiency of intersections and the traffic conditions of intersections. Especially, with the development of intelligent transportation systems (ITS) in recent years, obtaining a more accurate intersection ability to carry out intelligent control and management at intersections has become an important demand for traffic management departments.

In 2016, Article 47 of the Road Traffic Safety Law of China stipulates that when a motor vehicle passes a crosswalk, it shall slow down; when a pedestrian is passing the crosswalk, the vehicle shall stop and yield; when a motor vehicle passes a road without traffic signals, it shall yield to the pedestrian. "Vehicles yield to pedestrians" has changed from an idea to a mandatory traffic regulation. The regulation further increases pedestrian's safety in crosswalk and intersection and also leads to some changes in the mechanism of pedestrian-vehicle interaction.

So far, there are four main methods to calculate the capacity of intersections: the conflict point method [2-4], the stop line method $[3,5]$, the method of Design Regulation of Urban Road Engineering [6], and the saturation flow rate method [7-11]. Among them, except that the first calculation method is only used for special phase-controlled intersections, the other three methods can be applied for 
general signalized intersection, and the stop line method and the method of Design Regulation of Urban Road Engineering are essentially the saturation flow rate methods [12]. Therefore, the saturation flow rate method is the main method to calculate the capacity of an intersection, which is obtained by multiplying the basic saturated flow rate of the traffic flow and some correction parameters that affect the traffic flow.

In order to obtain a more accurate capacity of signalized intersection, much research has been carried out on capacity modification. The factors considered mainly include the following aspects: vehicle type [13, 14], lane width [15], the impact of bus stop [16], driver's behavior [17], lane utilization [18], the impact of short lane [19], left-turn traffic [7], right-turn traffic [7], access and leave traffic [20], the impact of interleaving area [21], the impact of pedestrian and nonmotor vehicle $[22,23]$, the impact of intelligent transport system [24], and weather and brightness conditions [25]. In the aspects above, the research method or theory of pedestrian's influence on traffic capacity mainly includes the following categories.

(a) Gap acceptance theory [22]: it is applied to the research of pedestrian and nonmotor vehicle on the traffic capacity of intersection by some scholars. However, the limitation of this theory is that it only takes the distance or time between pedestrian and vehicle as the consideration to distinguish the priority of vehicle and pedestrian to pass, with too few factors considered.

(b) Simulation model: at present, Legion, TransModer, PTV, and some other traffic simulation software have added pedestrian module. Based on the Vissim simulation model, Chen et al. [26] studied the sensitivity of intersection capacity to mean flow of pedestrians and nonmotor vehicles under mixed traffic conditions. However, computer simulation runs based on certain models (e.g., vehicle following model and social force model for pedestrian traffic simulation), which have their own limitations.

(c) Saturation flow rate correction method: it takes the pedestrian arrival rate as an independent variable and establishes a modified calculation model reflecting the interference of pedestrians (by studying the proportion of travel time available to pedestrians and vehicles in the conflict zone or the relationship between pedestrians and saturated headway [23] of traffic flow). However, due to the lack of discussion on the interaction mechanism between single pedestrian and vehicle, the calculation model established by the current study cannot adapt to the changes of some factors in the traffic environment (e.g., the promulgation of the new traffic regulations of "vehicles yield to pedestrians").

At crosswalk, the interaction between pedestrian and vehicle is affected by many factors. In this aspect, scholars have conducted a lot of research, mainly from the following three aspects: (a) Pedestrian factors: Mcmahon et al. [27] found that drivers are less likely to stop for black and male pedestrians on marked crosswalks; the research of Bourquin et al. [28] shows that some behaviors of pedestrians have an effect on the yield behavior of drivers (e.g., when a pedestrian is crossing the street, the action of raising hands can increase the probability of the drivers' yield rate); Zheng et al.'s [29] research shows that drivers are less likely to yield to pedestrians who do not obey traffic laws.

(b) Vehicle factors: driver is the decision-maker of a yield behavior. Because it is difficult to quantify the research, the current research on vehicles mainly focuses on the number of vehicles and driving speed. The research of Dou and Gou [30] shows that the yielding rate decreases with the increase of vehicle flow; studies by Stapleton et al. [31] and Sucha et al. [32] show that the yielding rate of vehicles with higher speed is less than that of vehicles with lower speed when they meet pedestrians crossing the street.

(c) Traffic environment factors: traffic environment includes road geometry, traffic control, and natural conditions. Stapleton et al. [31] studied the effect of adding auxiliary equipment at intersections on the yielding rate. The results show that the yielding rate would be significantly increased at intersections with Rectangular Rapid Flashing Beacons (RRFBs) equipment. Kay et al. [33] found that the difference of road width has an effect on driver's decisionmaking, Houten et al. [34] studied the impact of traffic law enforcement on the yielding rate. The results show that the yielding rate increases with the strengthening of traffic law enforcement, and after a period of traffic law enforcement in a certain area, if the traffic law enforcement is not continued, the yielding rate in this area will still maintain at a certain level.

The research above is of great significance for the study of the pedestrian-vehicle interaction process. However, most of the current theories and methods about pedestrian-vehicle interaction cannot adapt to the changes of the pedestrian-vehicle interaction mechanism brought by the changes of traffic regulations.

Based on the analysis of previous studies, the following two topics are urgently needed. One is to study the pedestrian-vehicle interaction mechanism under the combined influence of the new traffic regulations and important traffic environment factors (e.g., vehicle characteristics, pedestrian characteristics, and traffic conditions). The other considers the relevant impact of the pedestrian-vehicle interaction mechanism (e.g., intersection capacity, signal timing, and level of service (LOS)). Research on the impact of pedestrians on traffic capacity has received considerable attention. However, there has been little discussion about the capacity modification considering pedestrian-vehicle interaction.

The primary objective of this study is to propose a capacity evaluation method for the exclusive right-turn lane in 
signalized intersections to improve the assessment systems for intersections. The proposed PV-RTC model will be used (a) as a reference for intersection capacity estimation and service level evaluation when pedestrian-vehicle interaction is considered and (b) to provide traffic managers with more accurate information about signalized intersections to facilitate their formulation of traffic management measures. This research contributes to a more accurate capacity value of exclusive right-turn lane under the influence of pedestrians. This study mainly makes three contributions.

(a) Based on the quantitative method, the main factors influencing the interaction between pedestrians and vehicles are summarized. After that, a pedestrianvehicle interaction (PVI) model is proposed based on the logit model and static games theory of incomplete information.

(b) Based on the basic idea of the stop line method, an exclusive right-turn lane capacity model considering the pedestrian-vehicle interaction (PV-RTC) model is established. And then, this paper analyzes the influence of traffic environment change on the safety and efficiency of pedestrian-vehicle interaction and puts forward suggestions for improving traffic safety according to the sensitivity analysis results of the PVRTC model.

(c) The pedestrian-vehicle interaction microscopic simulation model is established based on cellular automata, and the validity of the proposed PV-RTC model under low pedestrian flow cases is verified by the microscopic simulation model after real data calibration.

The structure of this dissertation is as follows: the first section gives an introduction on the quantitative evaluation method of the PV-RTC model; the second section is concerned with the methodology. In this part, the PVI model is driven and the PV-RTC model is established; the next two sections are sensitivity analysis and model validation, which are established to clarify the proposed mechanism of the PVRTC model; and conclusion are made in the last section.

\section{Problem Description}

\subsection{Capacity Model Considering Pedestrian-Vehicle} Interaction. Pedestrian factor is a critical consideration in the capacity estimation of the exclusive right-turn lane in this paper. When pedestrian and vehicle meet at crosswalk, there is no information exchange between them, and pedestrian (vehicle) will not be able to accurately determine whether the vehicle (pedestrian) passes or not. Therefore, the essence of the decision-making made by driver and pedestrian is discrete choice behavior, and their choices are independent of each other. If the interaction mode between pedestrians and vehicles is not vehicles yield to pedestrians or pedestrians yield to vehicles, then there will be a game process of incomplete information between them in the next moment. After making clear the process of pedestrianvehicle interaction, the exclusive right-turn capacity can be obtained by taking this process into account in the calculation method of capacity. The layout of construction for the PV-RTC model is shown as Figure 1.

2.2. Parameter Description. To facilitate the model presentation, the key notations used hereafter are summarized in Table 1.

2.3. Mathematical Description. In this paper, a single cycle is divided into pedestrian-vehicle interaction time and nonpedestrian-vehicle interaction time, and the number of vehicles passing through each period of time is represented by letters a and $b$, respectively. According to the pedestrian-vehicle interaction process, it can be concluded that the number of vehicles passing through the lane during pedestrian-vehicle interaction time is related to the pedestrian arrival rate, probabilities of different pedestrian-vehicle interaction situations, and their interaction time and the number of vehicles passing through the lane during nonpedestrian-vehicle interaction time is related to the pedestrian arrival rate and probabilities of different pedestrian-vehicle interaction situations. In this paper, it is assumed that pedestrian arrival and vehicle arrival obey Poisson distribution, respectively, and all pedestrians obey the traffic laws.

Figure 2 is the block diagram of the PV-RTC model. Through the PVI model and its evolution model, the main parameters $\left(P_{m}\right)$ of the PV-RTC model can be obtained, and then the final capacity value can be obtained through the stop line method. In formula (1), $3600 / h_{t}$ is a formula for calculating the exclusive right-turn lane capacity based on the stop line method, where $h_{t}(\mathrm{~s})$ is the average headway of the right-turn traffic flow when there is no pedestrian influence $\left(h_{t}\right.$ can be obtained by the measured data):

$$
\begin{aligned}
C_{r} & =\frac{3600}{h_{t}}=\frac{3600}{c} \frac{c}{h_{t}} \longrightarrow \text { pedestrians } \frac{3600}{c}(a+b), \\
a & =\Phi_{1}\left(P_{m}, t_{m}, q_{m}\right), \\
b & =\Phi_{2}\left(P_{n}, q_{p}\right) .
\end{aligned}
$$

2.4. Capacity Model. In this section, the PVI model based on the logit model and static games theory of incomplete information is established, and then the PV-RTC model is proposed.

\subsection{Pedestrian-Vehicle Interaction Model. When a motor} vehicle meets a pedestrian crossing the street, both sides will make a pass or not pass decision after a short judgment [35]. On the basis of previous studies, this section first summarizes the factors that affect vehicle's and pedestrian's decision-making when they meet, then through the correlation analysis method to analyze the relevance between factors affecting pedestrian's and vehicle's decision-making and yielding rate, through the principal component analysis (PCA) method to analyze the mutual independence between 


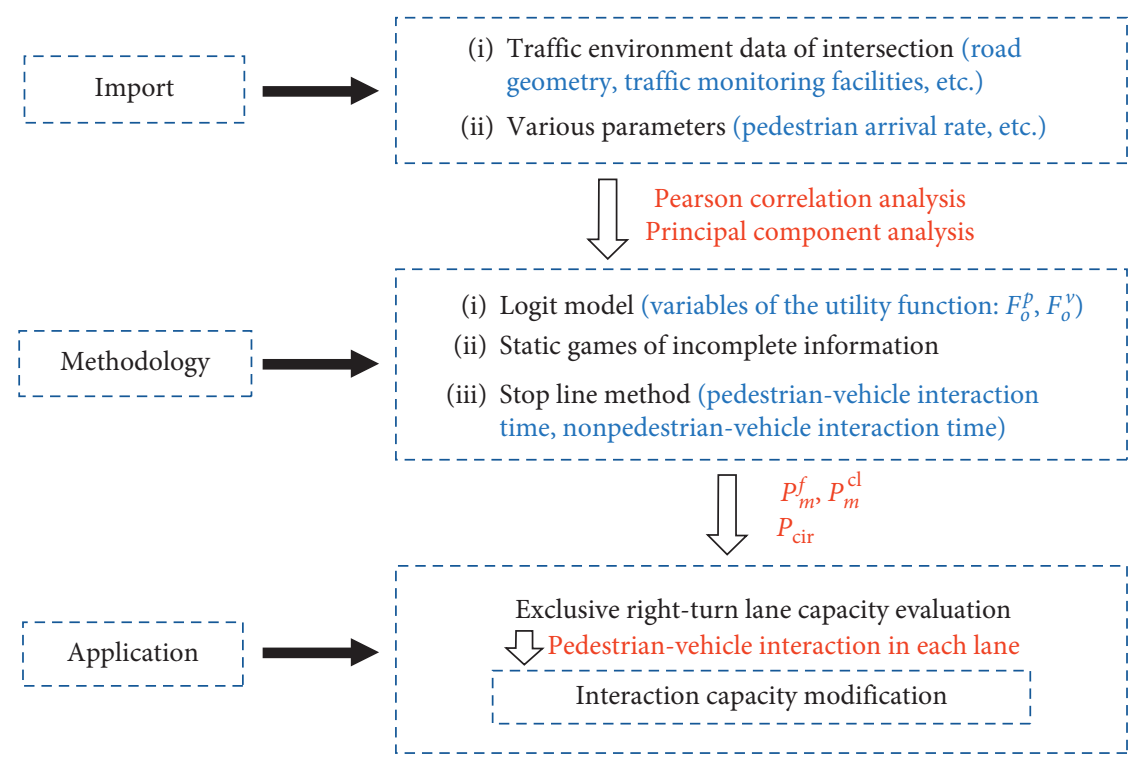

Figure 1: Layout of construction for the PV-RTC model.

factors affecting pedestrian's and vehicle's decision-making, and finally regarding the factors influencing pedestrian's and vehicle's decision-making as independent variables to establish the PVI model.

Due to the fact that the logit model can well describe the influence of the change of influencing factors (traffic environment, pedestrian flow, etc.) on the decision-making of pedestrian and vehicle and it is one of the most widely used discrete choice models, this paper establishes the PVI model based on the logit model.

2.6. Pedestrian's Decision-Making Model. When pedestrian and vehicle meet at crosswalk, the pedestrian will make a decision with the maximum utility for them (wait or cross). For different pedestrian-vehicle interaction processes, the utility corresponding to waiting and crossing is different, so pedestrian's decision-making is also different. The logit model of pedestrian's decision-making is as follows:

$$
P_{p}(i)=\frac{\exp U_{i}^{p}}{\sum_{i=1}^{2} \exp U_{i}^{p}} .
$$

Through Pearson's correlation analysis and principal component analysis, the principal component expressions for pedestrian's decision-making are as follows:

$$
F_{\alpha}^{p}=\sum_{j} k_{j}^{p \alpha} x_{j}^{\alpha}
$$

Definition of utility function: taking the principal components influencing pedestrian's decision-making as the independent variables of utility function, the formula is given as

$$
U_{i}^{p}=\sum_{\alpha} \theta_{\alpha}^{i} F_{\alpha}^{p}+a_{i}
$$

The model coefficients are regressed according to the specific traffic environment. Firstly, substitute the standardized original data into (5) to obtain the principal components $F_{\alpha}^{p}$ that affect pedestrian's decision-making and then regress the weight coefficients through $F_{\alpha}^{p}$ and the measured probability of pedestrian's decision-making.

2.7. Vehicle's Decision-Making Model. Similarly, when pedestrian and vehicle meet at crosswalk, the driver will make a decision with the maximum utility for them (yield or not). For different pedestrian-vehicle interaction processes, the utility corresponding to yielding and passing is different, so vehicle's decision-making is also different. The logit model of vehicle's decision-making is as follows:

$$
P_{v}(i)=\frac{\exp U_{i}^{v}}{\sum_{i=1}^{2} \exp U_{i}^{v}} \text {. }
$$

Through Pearson's correlation analysis and principal component analysis, the principal component expressions for vehicle's decision-making are as follows:

$$
F_{\gamma}^{v}=\sum_{r} k_{r}^{v \gamma} y_{r}^{\gamma}
$$

Definition of utility function: taking the principal components influencing vehicle's decision-making as the independent variable of utility function, the formula is given as

$$
U_{i}^{v}=\sum_{\beta} \mu_{\beta}^{i} F_{\beta}^{v}+b_{i} .
$$

In the section above, the pedestrian-vehicle interaction model is established. Through the model, the probabilities of the following four situations [35] (see Table 2) can be obtained, respectively: (1) vehicles yield to pedestrians and simultaneously pedestrians yield to vehicles, (2) vehicles yield to pedestrians, (3) pedestrians yield to vehicles, and (4) vehicle does not yield to pedestrian and simultaneously pedestrian does not yield to vehicle. For situations (2) and 
TABLE 1: The key notations.

$h_{t}$ : saturation headway under ideal condition $(\mathrm{s})$;

$\theta_{\tau}, \mu_{\tau}$ : weight coefficients of utility function of the logit model;

$U_{i}^{\mathrm{p}}, U_{i}^{\mathrm{v}}:$ utility function of pedestrian/vehicle;

$F_{\alpha}^{p}, F_{\gamma}^{v}$ : principal component $\alpha(\gamma)$ affecting pedestrian's (vehicle's) decision-making;

$X_{\varepsilon}, Y_{\varepsilon}:$ standardized variables affecting pedestrian's (vehicle's) decision-making;

$P_{p}^{\text {cro }}(i), P_{v}^{\text {cro }}(i)$ : probability of pedestrian's (vehicle's) choosing to cross $(i=1)$ or not $(i=2)$ at crosswalk cro;

$V_{v}$ : average speed of traffic flow in the exclusive right-turn lane $(\mathrm{km} / \mathrm{h})$;

$W_{\text {dis }}$ : distance between vehicle and pedestrian $(\mathrm{m})$;

$\psi$ : payoff function;

$S_{\omega}^{\delta}$ : security payoff of pedestrian or vehicle under situation $\delta$;

$E_{\omega}^{\delta}$ : time payoff of pedestrian or vehicle under situation $\delta$;

$V_{p}^{\delta}, V_{\gamma}^{\delta}$ : the speed of pedestrian (vehicle) crossing the exclusive right-turn lane under situation $\delta$;

$T_{p}^{\delta}, T_{v}^{\delta}:$ the time of pedestrian (vehicle) crossing the exclusive right-turn lane under situation $\delta$;

$E_{p 1}, E_{p 2}$ : pedestrian's decision-making payoff without the influence of vehicles;

$E_{v 1}, E_{v 2}$ : vehicle's decision-making payoff without the influence of pedestrians;

$\bar{E}_{p}, \bar{E}_{v}$ : pedestrian's (vehicle's) decision-making payoff under the influence of vehicles (pedestrians);

$x_{\delta}, y_{\delta}$ : probability of pedestrian (vehicle) choosing to cross or not in pedestrian-vehicle interaction evolutionary stage;

$\chi, \tau$ : weight coefficients of payoff function;

$C_{r}$ : exclusive right-turn lane capacity $(\mathrm{veh} / \mathrm{h})$;

$P_{\text {cir }}$ : the probability of different pedestrian-vehicle interaction situations;

c: cycle length (s);

$t_{\mathrm{g} j}^{\mathrm{cl}}$ : the total time for pedestrians to cross the crosswalk cl in each cycle (s);

$t_{\mathrm{gk}}^{t_{\mathrm{g}}}:$ the total time for pedestrians to cross the crosswalk $f$ in each cycle (s);

$t_{l}^{\mathrm{r}}$ : the total time for pedestrians to cross the crosswalks $f$ and cl simultaneously in each cycle (s);

$C_{\text {cir }}^{f}, C_{\text {cir }}^{\mathrm{cl}}$ : exclusive right-turn lane capacity under the influence of crosswalk cl or $f(\mathrm{veh} / \mathrm{h})$;

$\mathrm{C}_{3}$ : exclusive right-turn lane capacity without the influence of pedestrians $(\mathrm{veh} / \mathrm{h})$;

$k_{1}^{\mathrm{cl}}, k_{1}^{f}$ : bidirectional pedestrian influence coefficients at crosswalk $f$ or cl;

$k_{2}$ : average number of pedestrians per pedestrian cluster (ped);

$k_{3}^{\mathrm{cl}}, k_{3}^{f}$ : capacity model coefficients at crosswalk $f$ or $\mathrm{cl}$ (change with the number of pedestrians);

$k_{4}^{\mathrm{cl}}, k_{4}^{f}$ : overlapping coefficients of bidirectional pedestrian-vehicle interaction;

$t_{1}^{\text {crol }}, t_{2}^{\text {crol }}$ : the crossing time of pedestrians accumulated during red time in two different directions ( 1 or 2$)$ and crosswalks $(f$ or $\mathrm{cl})$ under situation $1(\mathrm{~s})$;

$t_{1}^{\text {cro2 }}, t_{2}^{\text {cro2 }}$ : the crossing time of pedestrians accumulated during red time in two different directions ( 1 or 2$)$ and crosswalks $(f$ or $\mathrm{cl})$ under situation $2(\mathrm{~s})$;

$t_{p}^{\text {cro1 }}$ : the maximum of $t_{1}^{\mathrm{crol}}, t_{2}^{\mathrm{cro1}}(\mathrm{s})$;

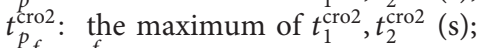

$\stackrel{Q}{p 1}_{p 1}^{f}, Q_{p 2}^{f}$ : pedestrian arrival rate at crosswalk $f$ in direction 1 or $2(\mathrm{ped} / \mathrm{c})$;

$Q_{p 1}^{\mathrm{cl}}, Q_{p 2}^{\mathrm{c}}:$ pedestrian arrival rate at crosswalk $\mathrm{cl}$ in direction 1 or $2(\mathrm{ped} / \mathrm{c})$;

$\mu_{f}, \mu_{\mathrm{cl}}$ : the proportion of accumulated pedestrians in the number of pedestrians arriving in each cycle;

$A$ : area occupied by single pedestrian $\left(\mathrm{m}^{2}\right)$;

$d$ : the width of the crosswalk (m);

$L$ : the width of the exclusive right-turn lane $(\mathrm{m})$;

$v_{p}$ : pedestrian crossing speed $(\mathrm{m} / \mathrm{s})$;

$P_{m}^{f}, P_{m}^{\mathrm{cl}}$ : pedestrian-vehicle interactive probability of situation $m$ at crosswalk $f$ or cl;

$t_{m}$ : pedestrian-vehicle interaction time of situation $m(\mathrm{~s})$;

$q_{p}, q_{p}^{\text {cl }}$ : pedestrian arrival rate at crosswalk $f$ or $\mathrm{cl}(\mathrm{ped} / \mathrm{c})$;

$q_{p 1}^{\text {cro }}, q_{p 2}^{\text {cro }}$ : pedestrian arrival rate in direction 1 or 2 at crosswalk cro $(\mathrm{ped} / \mathrm{c})$;

$\operatorname{myr}_{\mathrm{cl}}, \operatorname{myr}_{f}$ : yielding rate of crosswalk $f$ or cl;

$t_{v}^{\mathrm{cl}}, t_{v}^{f}$ : the time spent on vehicles passing at the early stage of pedestrian green time (s);

$\varphi_{v}^{f}, \varphi_{v}^{\mathrm{cl}}$ : the number of vehicles passing at the early stage of pedestrian green time (veh);

$Q_{v}^{r-t}$ : vehicle arrival rate of the exclusive right-turn lane (veh/h);

$v_{n}$ : the speed of vehicle in the cellular automata model (acceleration: $a$ );

$x_{n}$ : the position of vehicle in the cellular automata model;

$v_{i, j}$ : the speed of pedestrian in the cellular automata model;

$d_{i, j}, d_{i, j}^{j-1}, d_{i, j}^{j+1}$ : the number of cells in front of, in front of the left, and in front of the right of cell $(i, j)$.

(3), from the perspective of traffic efficiency, one side always passes first and the other side passes later and the interaction process is always completed at one time. However, for situations (1) and (4), the interaction process cannot be completed at one time, so it is necessary to further study the pedestrian-vehicle interaction process of situations (1) and (4). Due to the fact that, in both situations (1) and (4), pedestrian and vehicle are all in a state of mutual competition, in this section, the pedestrian-vehicle interaction evolutionary model based on static games of incomplete 


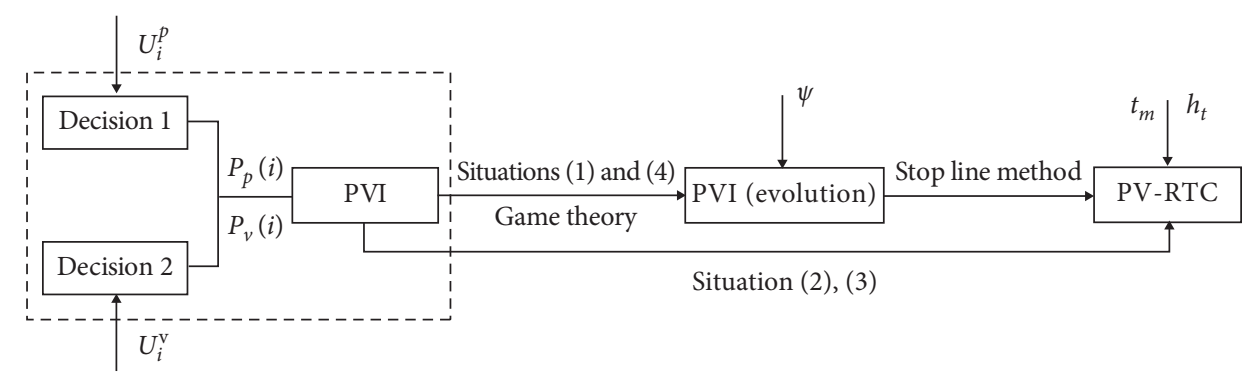

Figure 2: Block diagram of the PV-RTC model.

TABLE 2: Four situations of pedestrian-vehicle interaction and their corresponding probabilities.

\begin{tabular}{lc}
\hline Four situations & Probability of each situation \\
\hline Situation (1) & $P_{v}(2) P_{p}(2)$ \\
Situation (2) & $P_{p}(1) P_{v}(2)$ \\
Situation (3) & $P_{v}(1) P_{p}(2)$ \\
Situation (4) & $P_{v}(1) P_{p}(1)$ \\
\hline
\end{tabular}

information is established to explore the evolution results of situations (1) and (4).

According to static games of incomplete information theory, the game matrix of pedestrian and vehicle is firstly established, and then the state transition equation of pedestrian and vehicle is derived, respectively, through the game matrix. Finally, the probabilities of other interaction situations transferred from situations (1) and (4) are obtained, respectively. The process is as follows.

In situations (1) and (4), there will be conflict between pedestrian and vehicle. At this moment, both pedestrian and vehicle have two choices (pass or not). According to all possible combinations of choices, the pedestrian-vehicle interaction game matrix is established as shown in Table 3.

In order to obtain the respective payoff of pedestrian and vehicle under different combinations of choices, a payoff function considering the safety and benefits is introduced here, as shown in formula (10). In this payoff function, the safety $S_{\omega}^{\delta}$ is expressed by the speed expectation when the corresponding situation occurs, and the efficiency $E_{\omega}^{\delta}$ is expressed by the crossing time expectation when the corresponding situation occurs (see Table 4). Due to the difference of dimension between $S_{\omega}^{\delta}$ and $E_{\omega}^{\delta}$, they are firstly standardized by the range transformation method in the calculation, where $\chi, \tau$ are the weight coefficients of the payoff function, which are obtained through the questionnaire survey.

Payoff function:

$$
\psi=\chi S_{\omega}^{\delta}-\tau E_{\omega}^{\delta} .
$$

According to the game matrix, the equilibrium point of the game evolution between pedestrian and vehicle is derived as follows:

(a) Replicated dynamic equation of pedestrian:

The expected payoff of the pure strategy when pedestrian chooses to cross:
TABle 3: Game matrix.

\begin{tabular}{lcc}
\hline Pedestrian/vehicle & Pass & Yield \\
\hline Cross & $\left(a_{\delta}, b_{\delta}\right)$ & $\left(c_{\delta}, d_{\delta}\right)$ \\
Wait & $\left(e_{\delta}, f_{\delta}\right)$ & $\left(g_{\delta}, h_{\delta}\right)$ \\
\hline
\end{tabular}

$$
E_{p 1}=y_{\delta} a_{\delta}+\left(1-y_{\delta}\right) c_{\delta}
$$

The expected payoff of the pure strategy when pedestrian chooses to wait:

$$
E_{p 2}=y_{\delta} e_{\delta}+\left(1-y_{\delta}\right) g_{\delta}
$$

The expected payoff of the mixed strategy when pedestrian chooses to cross with probability of $x_{\delta}$ and chooses to wait with probability of $1-x_{\delta}$ :

$$
\bar{E}_{p}=x_{\delta} E_{p 1}+\left(1-x_{\delta}\right) E_{p 2} .
$$

From (11)-(13), the replicated dynamic equation that pedestrian chooses to cross is obtained:

$$
\begin{aligned}
F_{1}\left(x_{\delta}, y_{\delta}\right) & =\frac{\partial x_{\delta}}{\partial t}=x_{\delta}\left(E_{p 1}-\bar{E}_{p}\right)=x_{\delta}\left(1-x_{\delta}\right)\left(E_{p 1}-E_{p 2}\right) \\
& =x_{\delta}\left(1-x_{\delta}\right)\left(y_{\delta}\left(a_{\delta}+g_{\delta}-c_{\delta}-e_{\delta}\right)-\left(g_{\delta}-c_{\delta}\right)\right) .
\end{aligned}
$$

(b) Replicated dynamic equation of vehicle:

The expected payoff of the pure strategy when vehicle chooses to pass:

$$
E_{v 1}=x_{\delta} b_{\delta}+\left(1-x_{\delta}\right) f_{\delta}
$$

The expected payoff of the pure strategy when vehicle chooses to yield:

$$
E_{v 2}=x_{\delta} d_{\delta}+\left(1-x_{\delta}\right) h_{\delta} .
$$


TABLE 4: Security and time payoff of pedestrian and vehicle.

\begin{tabular}{lcc}
\hline & $S_{\omega}^{\delta}$ & $E_{\omega}^{\delta}$ \\
\hline$a_{\delta}$ & $P_{p}(1) P_{v}(2) V_{v}^{\delta}+P_{v}(1) P_{p}(2)\left(V_{p}^{\delta}+V_{v}^{\delta}\right)$ & $P_{p}(1) P_{v}(2) T_{p}^{\delta}+P_{v}(1) P_{p}(2)\left(T_{p}^{\delta}+T_{v}^{\delta}\right)$ \\
$b_{\delta}$ & $P_{2} V_{v}^{\delta}+P_{p}(1) P_{v}(2)\left(V_{p}^{\delta}+V_{v}^{\delta}\right)$ & $P_{2} T_{v}^{\delta}+P_{p}(1) P_{v}(2)\left(T_{p}^{\delta}+T_{v}^{\delta}\right)$ \\
$c_{\delta}$ & $V_{p}^{\delta}$ & $T_{p}^{\delta}$ \\
$d_{\delta}$ & $V_{p}^{\delta}+V_{v}^{\delta}$ & $T_{p}^{\delta}+T_{v}^{\delta}$ \\
$e_{\delta}$ & $V_{p}^{\delta}+V_{v}^{\delta}$ & $T_{p}^{\delta}+T_{v}^{\delta}$ \\
$f_{\delta}$ & $V_{v}^{\delta}$ & $T_{v}^{\delta}$ \\
$g_{\delta}$ & $P_{p}(1) P_{v}(2) V_{p}^{\delta}+P_{v}(1) P_{p}(2)\left(V_{p}^{\delta}+V_{v}^{\delta}\right)$ & $P_{p}(1) P_{v}(2) T_{p}^{\delta}+P_{v}(1) P_{p}(2)\left(T_{p}^{\delta}+T_{v}^{\delta}\right)$ \\
$h_{\delta}$ & $P_{v}(1) P_{p}(2) V_{v}^{\delta}+P_{p}(1) P_{v}(2)\left(V_{p}^{\delta}+V_{v}^{\delta}\right)$ & $P_{v}(1) P_{p}(2) T_{v}^{\delta}+P_{p}(1) P_{v}(2)\left(T_{p}^{\delta}+T_{v}^{\delta}\right)$ \\
\hline
\end{tabular}

The expected payoff of the mixed strategy when vehicle chooses to pass with probability of $y_{\delta}$ and chooses to yield with probability of $1-y_{\delta}$ :

$$
\bar{E}_{v}=y_{\delta} E_{v 1}+\left(1-y_{\delta}\right) E_{v 2} \text {. }
$$

From (15)-(17), the replicated dynamic equation that vehicle chooses to pass is obtained:

$$
\begin{aligned}
F_{2}\left(x_{\delta}, y_{\delta}\right) & =\frac{\partial y_{\delta}}{\partial t}=y_{\delta}\left(E_{v 1}-\bar{E}_{v}\right)=y_{\delta}\left(1-y_{\delta}\right)\left(E_{v 1}-E_{v 2}\right) \\
& =y_{\delta}\left(1-y_{\delta}\right)\left(x_{\delta}\left(b_{\delta}+h_{\delta}-f_{\delta}-d_{\delta}\right)-\left(h_{\delta}-f_{\delta}\right)\right), \\
& \left\{\begin{array}{l}
\frac{\partial x_{\delta}}{\partial t}=0, \\
\frac{\partial y_{\delta}}{\partial t}=0, \\
x_{\delta}, y_{\delta} \in[0,1] .
\end{array}\right.
\end{aligned}
$$

All possible evolutionary equilibrium points:

$(0,0),(1,0),(0,1),(1,1),\left(\frac{h_{\delta}-f_{\delta}}{h_{\delta}-f_{\delta}+b_{\delta}-d_{\delta}}, \frac{g_{\delta}-c_{\delta}}{g_{\delta}-c_{\delta}+a_{\delta}-e_{\delta}}\right)$.

After the derivation above, five possible evolutionary equilibrium points are finally obtained. The practical meaning corresponding to $(0,0)$ is that for situation (1) or (4), the evolution decision of pedestrian and vehicle is neither passed, which is inconsistent with the reality, so it is excluded. The practical meaning corresponding to $(1,0)$ is that all pedestrians choose to cross and all vehicles choose to yield, to $(0,1)$ is that all pedestrians choose to wait and all vehicles choose to pass, and to (1, 1 ) is that all pedestrians choose to cross and all vehicles choose to pass, which are also inconsistent with the reality, so they are excluded. Therefore, the final evolutionary equilibrium point $\left(x_{\delta}, y_{\delta}\right)$ of pedestrians and vehicles in situations (1) and (4) is $\left(\left(h_{\delta}-f_{\delta}\right) /\right.$ $\left.\left(h_{\delta}-f_{\delta}+b_{\delta}-d_{\delta}\right),\left(g_{\delta}-c_{\delta}\right) /\left(g_{\delta}-c_{\delta}+a_{\delta}-e_{\delta}\right)\right)$.

Through the derivation above, all kinds of evolutionary situations and their corresponding probabilities during pedestrian-vehicle interaction process can be obtained (see Table 5).

2.8. Exclusive Right-Turn Lane Capacity Model. In the previous part, the PVI model based on the logit model and static games theory of incomplete information theory was established, through which the probability corresponding to 6 kinds of pedestrian-vehicle interaction situations can be obtained (see Table 5). In this part, based on the basic idea of the stop line method, the PV-RTC model will be proposed as the following formula:

$$
C_{r}=\sum_{\text {cir }} \frac{P_{\text {cir }}}{c}\left[\sum_{j} t_{g j}^{\mathrm{cl}} C_{\text {cir }}^{f}+\sum_{k} t_{g k}^{f} C_{\text {cir }}^{\mathrm{cl}}\right]+\frac{C_{3}}{c} \sum_{l} t_{l}^{\mathrm{fr}} .
$$

The capacity formula consists of two parts: the first part represents the exclusive right-turn lane capacity when there exists pedestrian influence in a signal period (the capacity of this part is the probability combination of two kinds of pedestrian-vehicle interaction situations: (a) at the beginning of the pedestrian green time, the vehicles first yield to the pedestrians accumulated during pedestrian red time, and then the vehicles pass through and (b) at the beginning of the pedestrian green time, some vehicles do not yield to the pedestrians accumulated during pedestrian red time. The accumulated pedestrians have no chance to cross the crosswalk until these vehicles pass, and finally the vehicle flow continues to pass) and the second part represents the exclusive right-turn lane capacity when there exists no pedestrian influence in a signal period (e.g., some four-phase signalized intersections).

The capacity calculation formulas in the two situations mentioned above are given as follows.

2.8.1. Situation 1. For each situation, due to the fact that the pedestrian-vehicle interaction process at crosswalk $f$ and crosswalk, respectively, cl is different while the pedestrianvehicle interaction time at the two crosswalks is complementary in single cycle, the right-turn lane capacity under the independent influence of the two crosswalks is calculated, respectively, and then the exclusive right-turn lane capacity in situation 1 could be obtained by combining the capacities of the two crosswalks according to the time proportion.

The right-turn lane capacity of crosswalk $f$ : 
TABLE 5: Six situations of pedestrian-vehicle interaction and their corresponding probabilities.

\begin{tabular}{lcc}
\hline Six situations & Probability of each situation & Interaction time of each situation \\
\hline Situation (2) & $P_{p}(1) P_{v}(2)$ & $t_{1}$ \\
Situation (1) $\longrightarrow$ situation (2) & $P_{p}(2) P_{v}(2) x_{1}$ & $t_{2}$ \\
Situation (4) $\longrightarrow$ situation (2) & $P_{p}(1) P_{v}(1) x_{2}$ & $t_{3}$ \\
Situation (3) & $P_{v}(1) P_{p}(2)$ & $t_{4}$ \\
Situation (1) $\longrightarrow$ situation (3) & $P_{p}(2) P_{v}(2) y_{1}$ & $t_{5}$ \\
Situation (4) $\longrightarrow$ situation (3) & $P_{p}(1) P_{v}(1) y_{2}$ & $t_{6}$ \\
\hline
\end{tabular}

$$
\begin{aligned}
C_{\text {cir } 1}^{f} & =\frac{3600}{c}\left[\frac{c-k_{1} t_{p}^{f 1}-k_{3}^{f} \sum_{m=1}^{6} P_{m}^{f} t_{m}\left(\left(q_{p}^{f}-\mu_{f} q_{p 1}^{f}-\mu_{f} q_{p 2}^{f}\right) / k_{2}\right)}{t_{g}}+\frac{k_{4}\left(q_{p}^{f}-\mu_{f} q_{p 1}^{f}-\mu_{f} q_{p 2}^{f}\right) \sum_{n=1}^{3} P_{n}^{f}}{k_{2}}\right] \\
t_{1}^{f 1} & =\frac{\left(\left(Q_{p 1}^{f} \mu_{f} A\right) / d\right)+L}{v_{p}} \\
t_{2}^{f 1} & =\frac{\left(\left(Q_{p 2}^{f} \mu_{f} A\right) / d\right)+L}{v_{p}} \\
t_{p}^{f 1} & =\max \left(t_{1}^{f 1}, t_{2}^{f 1}\right) .
\end{aligned}
$$

The coefficients of the PV-RTC model are as follows:

$$
\begin{aligned}
& k_{1}^{f}=1+\rho_{1} \min \left(q_{p 1}^{\mathrm{fi}}, q_{p 2}^{\mathrm{fi}}\right), \\
& k_{1}^{\mathrm{cl}}=1+\rho_{1} \min \left(q_{p 1}^{\mathrm{cli}}, q_{p 2}^{\mathrm{cli}}\right), \\
& k_{3}^{f}=\rho_{3}^{1} q_{\mathrm{pz}}^{f} e^{-\rho_{3}^{2}}, \\
& k_{3}^{\mathrm{cl}}=\rho_{3}^{1} q_{\mathrm{pz}}^{c l} e^{-\rho_{3}^{2}}, \\
& k_{4}^{f}=\left(1-\rho_{4} \operatorname{myr}\left(\frac{q_{p}^{f}-\mu_{f} q_{p 1}^{f}-\mu_{f} q_{p 2}^{f}}{k_{2}}\right)\right), \\
& k_{4}^{\mathrm{cl}}=\left(1-\rho_{4} \operatorname{myr}\left(\frac{q_{p}^{c l}-\mu_{\mathrm{cl}} q_{p 1}^{c l}-\mu_{\mathrm{cl}} q_{p 2}^{c l}}{k_{2}}\right)\right) .
\end{aligned}
$$

$$
\begin{aligned}
C_{\mathrm{cir} 1}^{\mathrm{cl}} & =\frac{3600}{c}\left[\frac{c-k_{1} t_{p}^{\mathrm{cl} 1}-k_{3}^{\mathrm{cl}} \sum_{m=1}^{6} P_{m}^{\mathrm{cl}} t_{m}\left(\left(q_{p}^{\mathrm{cl}}-\mu_{\mathrm{cl}} q_{p 1}^{\mathrm{cl}}-\mu_{\mathrm{cl}} q_{p 2}^{\mathrm{cl}}\right) / k_{2}\right)}{t_{g}}+\frac{k_{4}\left(q_{p}^{\mathrm{cl}}-\mu_{\mathrm{cl}} q_{p 1}^{\mathrm{cl}}-\mu_{\mathrm{cl}} q_{p 2}^{\mathrm{cl}}\right) \sum_{n=1}^{3} P_{n}^{\mathrm{cl}}}{k_{2}}\right] \\
t_{1}^{\mathrm{cl} 1} & =\frac{\left(Q_{p 1}^{\mathrm{cl}} \mu_{\mathrm{cl}} A\right) / d+L}{v_{p}}, \\
t_{2}^{\mathrm{cl} 1} & =\frac{\left(Q_{p 2}^{\mathrm{cl}} \mu_{\mathrm{cl}} A\right) / d+L}{v_{p}} \\
t_{p}^{\mathrm{cl} 1} & =\max \left(t_{1}^{\mathrm{cl} 1}, t_{2}^{\mathrm{cl} 1}\right) .
\end{aligned}
$$

Formula (21) consists of two parts, the first part represents the number of vehicles passing in a single cycle when there exists no pedestrian-vehicle interaction crosswalk $f$ (the cycle time is subtracted from the time spent on 8 types of pedestrian-vehicle interaction and the time spent on accumulated pedestrians crossing at the beginning stage of green time); The second part represents the number of vehicles passing in a single cycle when there exists pedestrian-vehicle interaction crosswalk $f$.

Similarly, the capacity formula of crosswalk $\mathrm{cl}$ and the two capacity formulas of situation 2 are given as follows.

The right-turn lane capacity of crosswalk cl: 
2.8.2. Situation 2. The right-turn lane capacity of crosswalk

$f:$

$$
\begin{aligned}
C_{\mathrm{cir} 2}^{f} & =\frac{3600}{c}\left[\frac{c-t_{v}^{f}-k_{1} t_{p}^{f 2}-k_{3}^{f} \sum_{m=1}^{6} P_{m}^{f} t_{m}\left(\left(q_{p}^{f}-\mu_{f} q_{p 1}^{f}-\mu_{f} q_{p 2}^{f}\right) / k_{2}\right)}{t_{g}}+\varphi_{v}^{f}+\frac{\left.k_{4}\left(q_{p}^{f}-\mu_{f} q_{p 1}^{f}-\mu_{f} q_{p 2}^{f}\right) \sum_{n=1}^{3} P_{n}^{f}\right]}{k_{2}}\right] \\
t_{1}^{f 2} & =\frac{\left(Q_{p 1}^{f} \mu_{f} A\right) / d+L}{v_{p}}, \\
t_{2}^{f 2} & =\frac{\left(Q_{p 2}^{f} \mu_{f} A\right) / d+L}{v_{p}} \\
t_{p}^{f 2} & =\max \left(t_{1}^{f 2}, t_{2}^{f 2}\right) .
\end{aligned}
$$

The right-turn lane capacity of crosswalk cl:

$$
\begin{aligned}
C_{\mathrm{ci} 2}^{\mathrm{cl}} & =\frac{3600}{c}\left[\frac{c-t_{v}^{\mathrm{cl}}-k_{1} t_{p}^{\mathrm{cl} 2}-k_{3}^{\mathrm{cl}} \sum_{m=1}^{6} P_{m}^{\mathrm{cl}} t_{m}\left(\left(q_{p}^{\mathrm{cl}}-\mu_{\mathrm{cl}} q_{p 1}^{\mathrm{cl}}-\mu_{\mathrm{cl}} q_{p 2}^{\mathrm{cl}}\right) / k_{2}\right)}{t_{g}}+\varphi_{v}^{\mathrm{cl}}+\frac{k_{4}\left(q_{p}^{\mathrm{cl}}-\mu_{\mathrm{cl}} q_{p 1}^{\mathrm{cl}}-\mu_{\mathrm{cl}} q_{p 2}^{\mathrm{cl}}\right) \sum_{n=1}^{3} P_{n}^{\mathrm{cl}}}{k_{2}}\right] \\
t_{1}^{\mathrm{cl} 2} & =\frac{\left(Q_{p 1}^{\mathrm{cl}} \mu_{\mathrm{cl}} A\right) / d+L}{v_{p}}, \\
t_{2}^{\mathrm{cl} 2} & =\frac{\left(Q_{p 2}^{\mathrm{cl}} \mu_{\mathrm{cl}} A\right) / d+L}{v_{p}}, \\
t_{p}^{\mathrm{cl} 2} & =\max \left(t_{1}^{\mathrm{cl} 2}, t_{2}^{\mathrm{cl} 2}\right) .
\end{aligned}
$$

\section{Sensitivity Analysis}

A numerical case study will be presented. Various factors are taken into account to investigate effects on the PV-RTC model. The following is a brief summary of the assumptions that have been made before conducting the simulation.

(a) The number of vehicle arrivals per cycle is a random variable with a known probability distribution. In the case of an isolated intersection, the Poisson distribution is employed. The number of vehicles arrivals in each cycle time obeys Poisson distribution.

(b) The pedestrian arrival in each cycle time obeys Poisson distribution.

(c) All pedestrians obey the traffic laws.

In this section, to illustrate the applicability of the proposed models, this study applies the control approach to the intersection, which has exclusive right-turn lane (each right-turn lane has two crosswalks at the intersection). The basic parameters are shown in Table 6.
3.1. Sensitivity to Pedestrian Flow. Figures 3(a) and 3(b) show the change of exclusive right-turn capacity under the change of the pedestrian arrival rate in direction 1 of crosswalk $f$. In Figure 3(a), calculations show the difference of average capacity between two adjacent curves is 34 and $25 \mathrm{veh} / \mathrm{h}$, respectively, and the difference of average capacity between two adjacent curves in Figure 3(b) is 9 and $10 \mathrm{veh} / \mathrm{h}$, respectively. The capacity difference between adjacent curves in Figure 3(a) is greater than that in Figure 3(b). A possible explanation for this phenomenon is as follows: the pedestrian green time of crosswalk $f$ is $90 \mathrm{~s}$ and that of crosswalk cl is $27 \mathrm{~s}$ in each cycle, when other conditions are certain, and pedestrians at crosswalk $f$ have a longer influence on rightturn traffic flow than that of crosswalk $\mathrm{cl}$, which means a longer pedestrian-vehicle interaction time of crosswalk $f$ and longer delay time to right-turn traffic flow. Figures 3(a) and 3(b) have the same pedestrian arrival rate but different green times (a longer time for crosswalk $f$ ) for pedestrians. Therefore, the capacity of crosswalk $f$ has a wider range of changes. 
TABLe 6: Parameters in sensitivity analysis.

\begin{tabular}{|c|c|}
\hline Parameter & Physical value \\
\hline \multicolumn{2}{|c|}{ Vehicles yield to pedestrians (field measured) } \\
\hline$t_{1}(\mathrm{~s})$ & 4.6 \\
\hline$t_{2}(\mathrm{~s})$ & 5.2 \\
\hline$t_{3}(\mathrm{~s})$ & 5.7 \\
\hline \multicolumn{2}{|c|}{ Pedestrians yield to vehicles (field measured) } \\
\hline$t_{4}(\mathrm{~s})$ & 3.7 \\
\hline$t_{5}(\mathrm{~s})$ & 4.8 \\
\hline$t_{6}(\mathrm{~s})$ & 5.1 \\
\hline \multicolumn{2}{|c|}{ The probability of different pedestrian-vehicle interaction situations } \\
\hline$P_{1}$ & $(0.95)$ \\
\hline$P_{2}$ & $(0.05)$ \\
\hline \multicolumn{2}{|c|}{ Two situations at intersection } \\
\hline$t_{v}^{\mathrm{cl}}(\mathrm{s})$ & 25 \\
\hline$t_{v}^{f}(\mathrm{~s})$ & 20.5 \\
\hline$\varphi_{v}^{\mathrm{cl}}(\mathrm{veh})$ & 4 \\
\hline$\varphi_{v}^{f}(\mathrm{veh})$ & 3 \\
\hline \multicolumn{2}{|c|}{ Parameters of the PV-RTC model } \\
\hline$k_{1}^{f}, k_{1}^{\mathrm{cl}}$ & $\rho_{1}=0.005$ \\
\hline$k_{2}$ & 1.3 \\
\hline$k_{3}^{f}, k_{3}^{\mathrm{cl}}$ & $\rho_{3}^{2}=-0.5806$ \\
\hline$k_{4}^{f}, k_{4}^{\mathrm{cl}}$ & $\rho_{4}=0.1$ \\
\hline \multicolumn{2}{|c|}{ Parameters of traffic environment } \\
\hline$A\left(\mathrm{~m}^{2}\right)$ & $0.75 * 0.75$ \\
\hline$c(\mathrm{~s})$ & 120 \\
\hline$t_{g}^{\mathrm{cl}}(\mathrm{s})$ & 27 \\
\hline$t_{g}^{f}(\mathrm{~s})$ & 90 \\
\hline$v_{p}(\mathrm{~m} / \mathrm{s})$ & 1.2 \\
\hline$t_{g}(\mathrm{~s})$ & 3.3 \\
\hline \multicolumn{2}{|c|}{ Parameters of the crosswalk geometry } \\
\hline$d(\mathrm{~m})$ & 2.75 \\
\hline$L(\mathrm{~m})$ & 4.0 \\
\hline
\end{tabular}
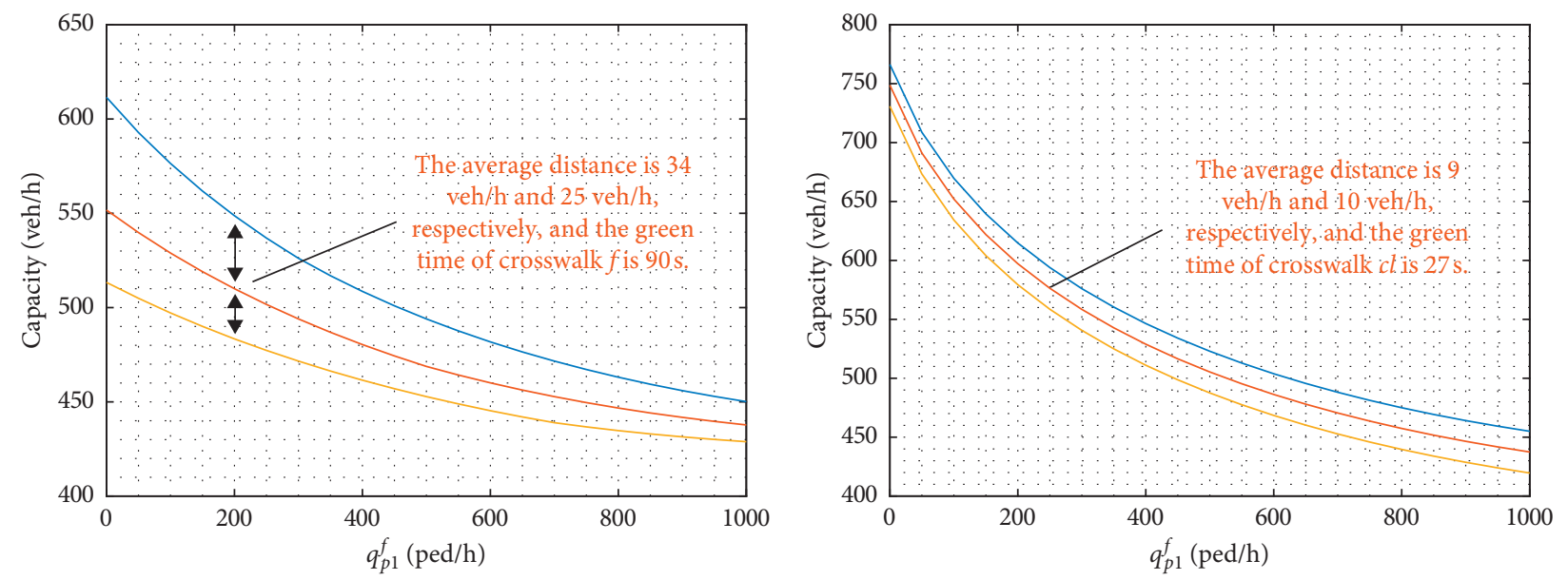

$$
\begin{aligned}
-q_{p 2}^{f} & =300(\mathrm{ped} / \mathrm{h}) \\
-q_{p 2}^{f} & =500(\mathrm{ped} / \mathrm{h}) \\
-q_{p 2}^{f} & =700(\mathrm{ped} / \mathrm{h})
\end{aligned}
$$$$
-q_{p 1}^{\mathrm{cl}}=300(\mathrm{ped} / \mathrm{h})
$$$$
-q_{p 1}^{\mathrm{cl}}=500(\mathrm{ped} / \mathrm{h})
$$$$
-q_{p 1}^{\mathrm{cl}}=700(\mathrm{ped} / \mathrm{h})
$$

(a)

(b)

Figure 3: Continued. 


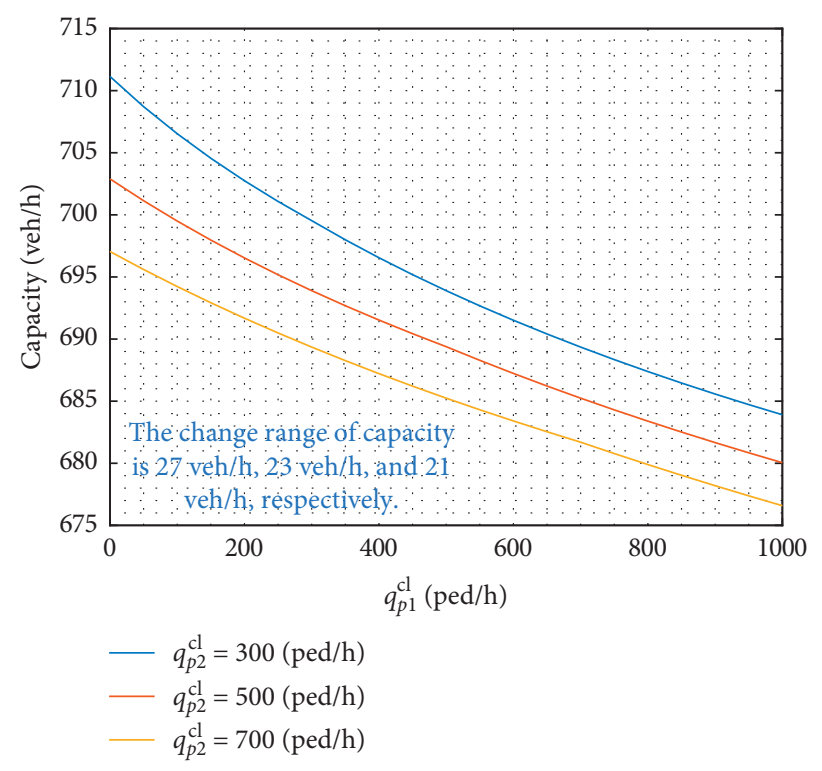

(c)

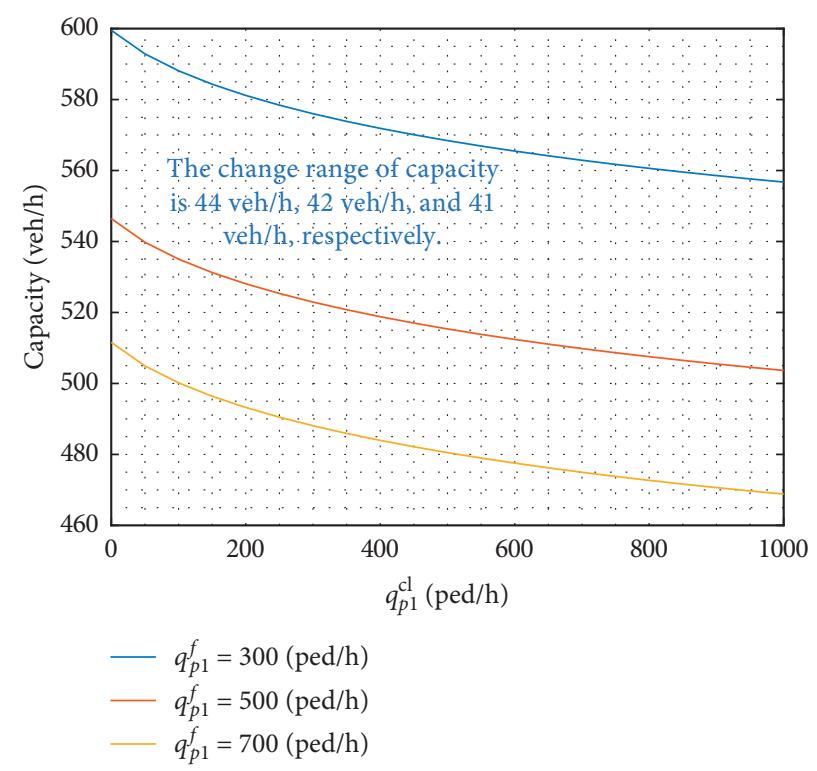

(d)

FIGURE 3: The sensitivity of capacity to the pedestrian arrival rate.

In addition, the average change range of capacity in Figure $3(\mathrm{a})$ is $24 \mathrm{veh} / \mathrm{h}$, and, accordingly, the change range in Figure 3(b) is $42 \mathrm{veh} / \mathrm{h}$. Figure 3(b) also shows a wider range of changes. A possible explanation is that the variables $q_{p 1}^{f}$, $q_{p 2}^{f}$ studied in Figure 3(a) are in the same crosswalk (crosswalk $f$ ), so pedestrians in direction 2 can take advantage of the crossing time in direction 1 to cross the lane; while the variables $q_{p 1}^{\rho}, q_{p 2}^{\mathrm{cl}}$ studied in Figure $3(\mathrm{~b})$ are in crosswalk cl and crosswalk $f$, respectively, under the same pedestrian arrival rate, the time effect of this situation on traffic flow is almost twice that of Figure 3(a).

To verify the conjecture above, Figures 3(c) and 3(d) present the influence of the change of the pedestrian arrival rate $(0-1000 \mathrm{ped} / \mathrm{h})$ at crosswalk $\mathrm{cl}$ on the exclusive rightturn lane capacity. It can be found that the capacity difference between adjacent curves in Figure 3(d) is greater than that of Figure 3(c) and the average change range of pedestrian capacity in Figure 3(d) is greater than that of Figure $3(\mathrm{c})$, which is consistent with the phenomena reflected in Figures 3(a) and 3(b). Therefore, it can be assumed that the results of sensitivity analysis are considered to be consistent with the actual situation.

3.2. Sensitivity to Yielding Rate. In practice, the probability of vehicle and pedestrian choosing to pass the lane or not is independent of each other [35]. The change of some factors in intersection will have different degrees of influence on the decision-making of pedestrian and vehicle. Therefore, it is necessary to analyze the probability combination of each decision-making of pedestrian and vehicle.

In Figure 4(a), when both $P_{p}^{f}(1)$ and $P_{v}^{f}(1)$ are close to 0 or 1 , the capacity reaches its minimum $(480-500 \mathrm{veh} / \mathrm{h})$; when $P_{p}^{f}(1)$ is close to 0 and $P_{v}^{f}(1)$ is close to 1 , the capacity reaches its maximum $(660-680 \mathrm{veh} / \mathrm{h})$. A possible explanation for the results might be that when both pedestrian and vehicle choose to yield or pass at the same time, pedestrian-vehicle interaction efficiency will be greatly reduced. This is because when vehicle and pedestrian meet in the conflict area and $P_{p}^{f}(1)$ and $P_{v}^{f}(1)$ are close to 0 , both sides make the decision not to pass. In this case, the delay time of traffic flow increases and the right-turn lane capacity decreases; when $P_{p}^{f}(1)$ and $P_{v}^{f}(1)$ are both close to 1 , although the vehicle makes the decision to pass when it meets a pedestrian, the pedestrian also chooses to pass. In this case, both sides enter the next decision-making moment, which increases the delay time of traffic flow and the vehicles capacity of the exclusive right-turn lane is reduced accordingly. When $P_{v}^{f}(1)$ is close to 1 and $P_{p}^{f}(1)$ is close to 0 and the pedestrian arrival rate $\left(q_{p 1}^{f}\right)$ in Figure $4(a)$ is only $300 \mathrm{ped} / \mathrm{h}$, the traffic flow is little affected by pedestrians; therefore the capacity reaches its maximum.

In Figure 4(b), $q_{p 1}^{f}=500 \mathrm{ped} / \mathrm{h}$. Compared with Figure 4(a), there is almost no difference between Figures $4(\mathrm{a})$ and $4(\mathrm{~b})$ in $P_{p}^{f}(1)$ and $P_{v}^{f}(1)$ corresponding to the maximum and minimum capacity. What is different is that the corresponding capacity of each pair of $P_{p}^{f}(1)$ and $P_{v}^{f}(1)$ reduced by about $100 \mathrm{veh} / \mathrm{h}$. A possible explanation for this phenomenon is that under certain other conditions, the increase of the pedestrian arrival rate not only increases the number of pedestrians crossing the street at the beginning of the pedestrians green time, but also increases the number of pedestrian-vehicle interactions, which increases the delay time of the traffic flow; therefore the capacity of the exclusive right-turn lane is reduced.

In addition, it can be found that the number of the maximum capacity points in Figure 4(b) increases and they are all concentrated in area $H\left(P_{v}^{f}(1)>0.7, P_{p}^{f}(1)<0.2\right)$, which 


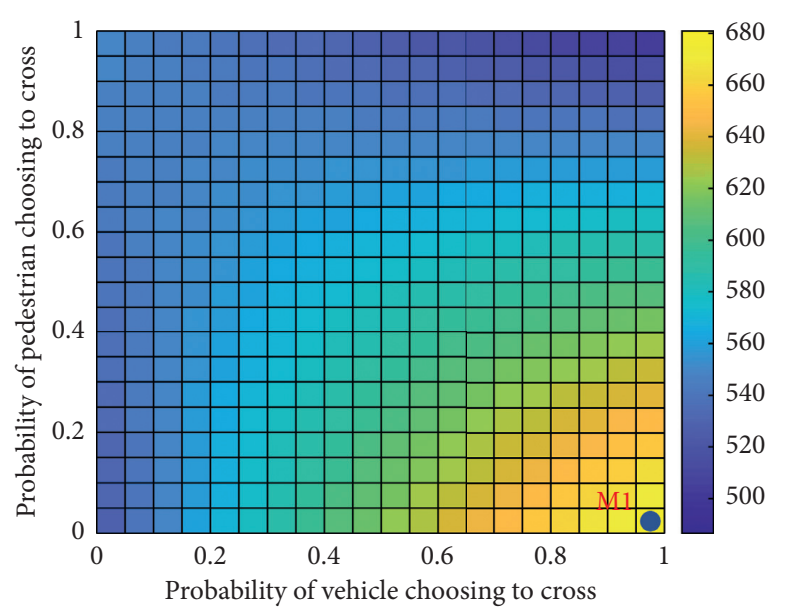

(a)

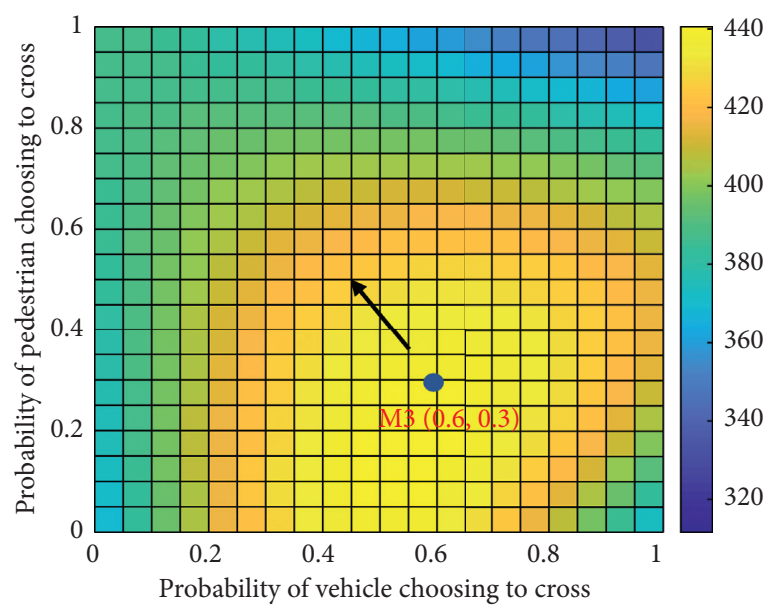

(c)

Figure 4: The sensitivity of capacity to $P_{p}^{f}(1)$ and $P_{v}^{f}(1)$. (d) $q_{p 1}^{f}=900(\mathrm{ped} / \mathrm{h})$.

indicates that when $q_{p 1}^{f}$ increases to $500 \mathrm{veh} / \mathrm{h}$, the capacity has little difference in these probability combinations (e.g., $P_{v}^{f}(1)=0.9, \quad P_{p}^{f}(1)=0.1 \quad$ (combination 1$) ; \quad P_{v}^{f}(1)=0.8$, $P_{p}^{f}(1)=0.2$ (combination 2$)$ ). A possible explanation is that compared with probability combination 2 , vehicles in probability combination 1 have a higher probability of pass, but the crossing rate of pedestrians is smaller. Those pedestrians with small crossing rate may choose to cross with a greater probability in the evolutionary decision-making stage, which has a greater impact on the traffic flow than probability combination 2. Therefore, in general, there is almost no difference in capacity between the two probability combinations.

In Figure 4(c), $q_{p 1}^{f}=900 \mathrm{ped} / \mathrm{h}$. It can be concluded that the increase in the pedestrian arrival rate continues to reduce the capacity of the right-turn lane. Compared with $q_{p 1}^{f}=500 \mathrm{ped} / \mathrm{h}$, the capacity values corresponding to each pair of probability combinations are reduced by about $90 \mathrm{veh} /$ h. In addition, different from $q_{p 1}^{f}=300 \mathrm{ped} / \mathrm{h}$ and $q_{p 1}^{f}=500 \mathrm{ped} / \mathrm{h}$, in this case, the maximum capacity points are no longer concentrated in the area where $P_{v}^{f^{\prime}}(1)$ is very large and $P_{p}^{f}(1)$ is very small, but move away from point $\mathrm{M} 2$, and

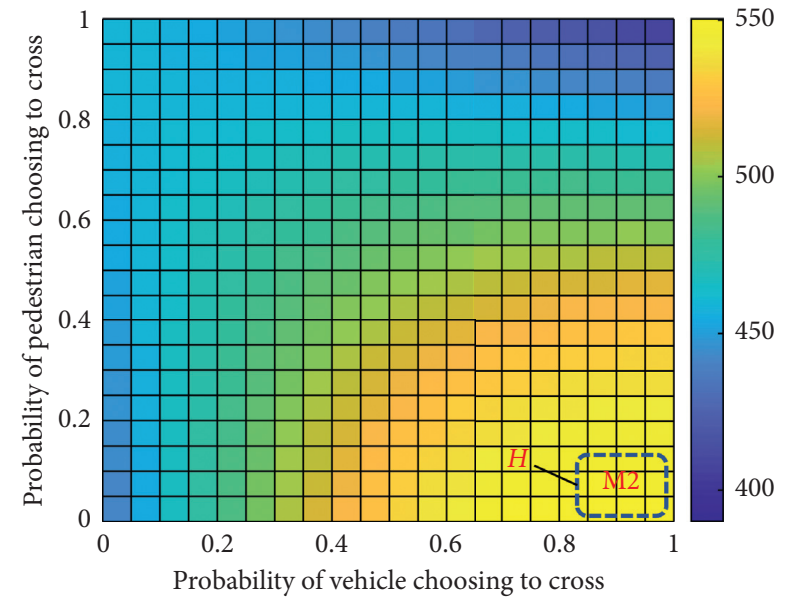

(b)

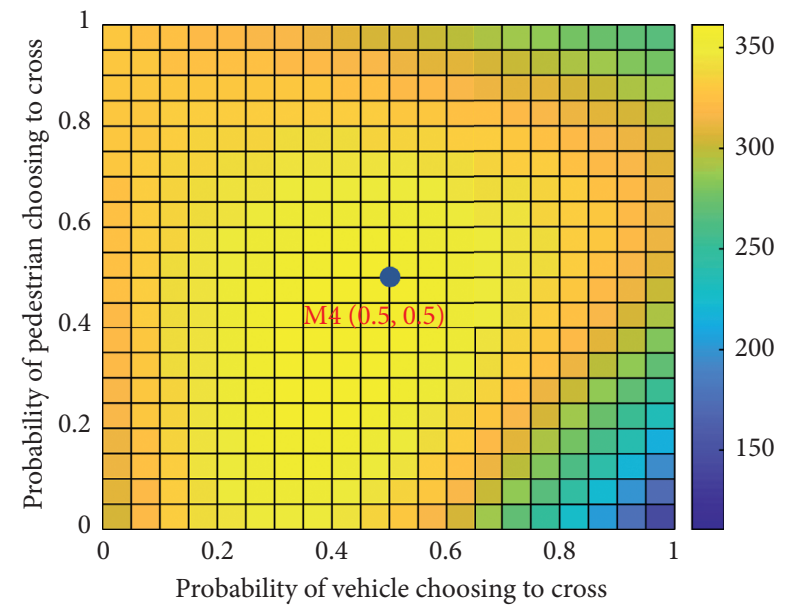

(d)

finally stop near the center of Figure 4(c), M3(0.6, 0.3). The traffic capacity around M3 is gradually decreasing. Analysis of this phenomenon is as follows: when the pedestrian arrival rate increases to a certain value, if $P_{v}^{f}(1)$ is still very large (more than 0.7 ) and $P_{P}^{f}(1)$ is still very small (less than 0.3 ), then the arriving pedestrians in each cycle will not be able to cross the street completely, and the detained pedestrians may have time impact on the traffic flow at the beginning stage of pedestrian green time or evolutionary decision-making stage, which decreases the capacity of the exclusive right-turn lane. Therefore, when the pedestrian arrival rate reaches 7004 ped/ $h$, the maximum point of capacity shifts.

Figure $4(\mathrm{~d})$ presents capacity change with the change of probability combination when $q_{p 1}^{f}=900 \mathrm{ped} / \mathrm{h}$. The change trend of Figure $4(\mathrm{~d})$ is consistent with that of Figure 4(c). When $P_{p}^{f}(1)$ and $P_{v}^{f}(1)$ are both 0.5 , the capacity reaches its maximum, which proves the rationality of the conjecture above on the phenomenon of Figure 4(c).

The research results above can be used for references in the efficiency and safety management during the pedestrianvehicle interaction process. 
(a) In efficiency aspect: because the traffic environment (traffic management facilities, road conditions, etc.) and traffic volume (pedestrians, vehicles, etc.) at a specific intersection are relatively stable in a certain period of time and the probabilities of different pedestrian-vehicle interaction situations in this intersection are also relatively stable. However, in this case, the pedestrian-vehicle interaction efficiency is not necessarily the highest (e.g., the right-turn lane capacity is not at its maximum). When the traffic management department is committed to improving the efficiency of pedestrian-vehicle interaction, they can obtain the optimal $P_{p}^{f}(1)$ and $P_{v}^{f}(1)\left(P_{p, m}^{f}(1)\right.$, $\left.P_{v, m}^{f}(1)\right)$ under any pedestrian arrival rate according to the methods above and then change the measured $P_{p}^{f}(1), P_{v}^{f}(1)$ by changing the traffic environment (e.g., install monitoring devices at crosswalks and add speed limit sign), so that they can reach their corresponding optimal value $\left(P_{p, m}^{f}(1), P_{v, m}^{f}(1)\right)$ in which the right-turn lane capacity reaches its maximum.

(b) In safety aspect: through the PVI model, the probability that pedestrian chooses to cross and vehicle chooses to cross $\left(P_{v}(1) P_{p}(1)\right)$ can be obtained. Because of the high probability of traffic accident in this situation, the probability can be used as the basis for traffic management departments to determine the safety level of the crosswalk. When is found is that the probability of such situation is relatively large; the probability can also be reduced by adjusting the traffic environment, so as to increase the safety level of pedestrian-vehicle interaction.

3.3. Sensitivity to Green Time Radio. In the sensitivity analysis of the pedestrian arrival rate, the influence of different pedestrian green times on the exclusive right-turn lane capacity has been preliminarily observed. In this section, the sensitivity of the green time ratio of the PV-RTC model will be analyzed.

It can be found that the general trend of Figures 5(a) and 5 (b) is that with the change of green time, the change of the exclusive right-turn lane capacity increases first and then decreases, and each curve corresponds to a maximum value. This is because for a given cycle time, under certain conditions (pedestrian arrival rate, probability of pedestrian (vehicle) choosing to cross, etc.), the optimal allocation of pedestrian green time in one cycle is obtained for each crosswalk. And this is why, in 5 different, cases the distance between the 5 curves gradually decreases and eventually converges to a point $\left(\mathrm{B}_{\kappa}\right)$.

In addition, compared with Figure 5(a), the maximum point of each curve in Figure 5(b) decreases and the optimal green time corresponding to the maximum point decreases (the green time ratio decreases). The decline rate of rightturn lane capacity in Figure 5(b) increases when the maximum capacity is reached, which is the result of the increase of the pedestrian arrival rate. When pedestrian green time of crosswalk $c$ reaches $120 \mathrm{~s}$, only crosswalk $c$ has an impact on the right-turn lane (the impact time is $120 \mathrm{~s}$ ). At this moment, the difference between the ordinates of B1, B2 reflects the difference of $q_{p 2}^{\mathrm{cl}}$ in the two figures.

Figures 5(c) and 5(d) present capacity change with the change of $P_{p}^{\mathrm{cl}}(1), P_{v}^{\mathrm{cl}}(1)$. The overall trend of Figures 5(c) and 5(d) is the same as that of Figures 5(a) and 5(b). When the pedestrian's green time of the crosswalk $\mathrm{cl}$ is 0 , only crosswalk $f$ has an impact on the right-turn lane (the impact time is $120 \mathrm{~s})$. At this moment, the difference of the coordinates of $D_{P}$ reflects the difference of $P_{p}^{\mathrm{cl}}(1), P_{v}^{\mathrm{cl}}(1)$, respectively, in the two figures. When the pedestrian green time of crosswalk $c$ reaches $120 \mathrm{~s}$, crosswalk $f$ has no impact on traffic flow. At this moment, only the crosswalk $c$ has an impact on the right-turn lane (the impact time is $120 \mathrm{~s}$ ), and both $P_{p}^{\mathrm{cl}}, P_{v}^{\mathrm{cl}}$ at crosswalk $\mathrm{cl}$ are 0.5 , so there is no difference in capacity in the two figures, which is consistent with the actual situation.

Further analysis of the maximum capacity points: in Figures 5(a)-5(d), the maximum point of each curve represents the maximum capacity of the right-turn lane under a certain pedestrian arrival rate. Therefore, theoretically, the maximum capacity of exclusive right-turn lane under any combination of pedestrian arrival rates and its corresponding green time ratio can be obtained, which provides a new idea for the optimal signal timing of intersections. For example, the capacity estimation method based on pedestrian-vehicle interaction can be used to obtain the optimal green time ratio of each lane under a certain pedestrian arrival rate and yielding rate, and then the optimal green time ratio of the intersection can be obtained according to the different demand degree of each lane for capacity (e.g., bus lane).

This kind of signal timing optimization method based on pedestrian-vehicle interaction has two merits ((a) take the demand degree of capacity of each lane into account and (b) fully consider the impact of pedestrians on each vehicle flow) and is applicable for some intersections with fixed cycle to optimize signal timing (e.g., green wave coordination control).

\section{Model Validation}

4.1. Pedestrian-Vehicle Interaction Model of Cellular Automata. Due to the limitations of Vissim and other microsimulation software that cannot adequately simulate the pedestrian-vehicle interaction process, therefore, by introducing the mutual interference and conflict rules of pedestrians and vehicles at intersections and coupling the NaSch model [36] describing vehicle flow and the Blue model [36] describing pedestrian flow, a pedestrian-vehicle interaction model of cellular automata in this section is established to describe the interaction process between pedestrians and vehicles in the exclusive right-turn lane.

For vehicle flow in the exclusive right-turn lane, the whole pedestrian-vehicle interaction process is equivalent to that of the vehicles passing through two near crosswalks. Therefore, in the pedestrian-vehicle interaction model of cellular automata, two mutually perpendicular crosswalks in the exclusive right-turn lane at intersections are regarded as 

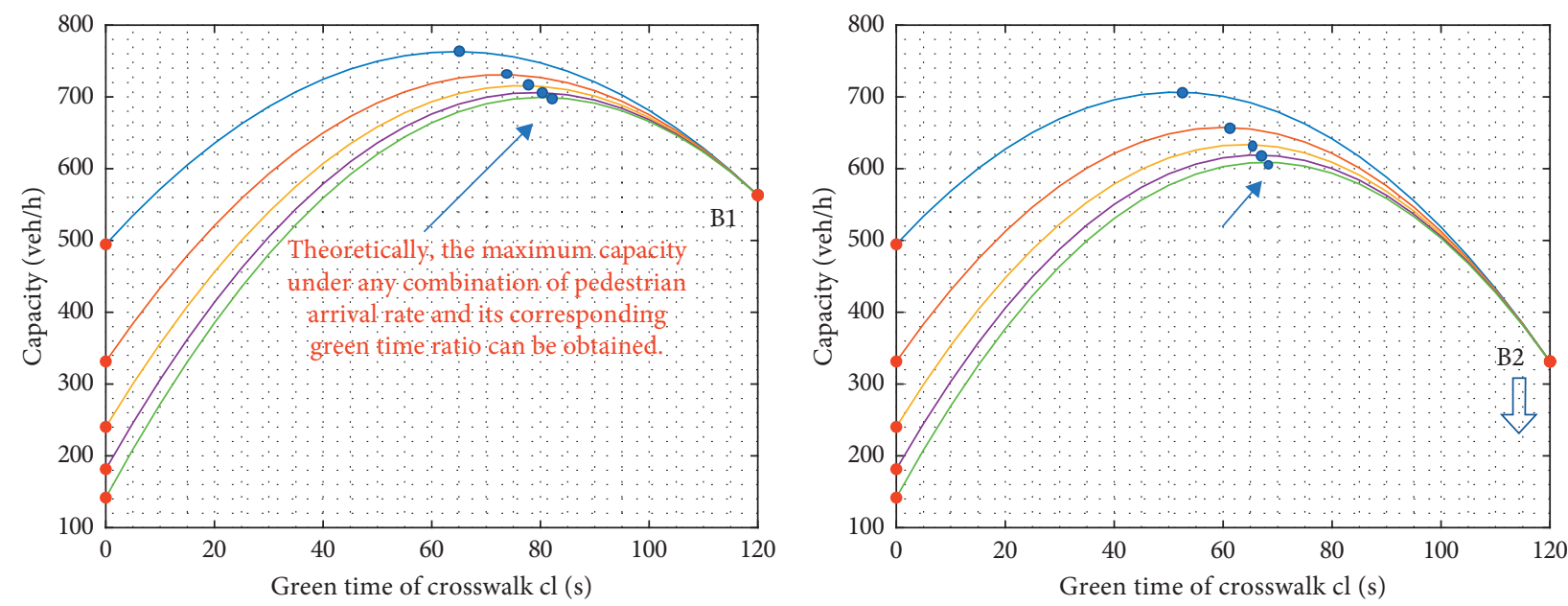

$$
\begin{aligned}
& -q_{p 1}^{f}=100(\mathrm{ped} / \mathrm{h}) \quad-q_{p 1}^{f}=700(\mathrm{ped} / \mathrm{h}) \\
& -q_{p 1}^{f}=300(\mathrm{ped} / \mathrm{h}) \quad-q_{p 1}^{f}=900(\mathrm{ped} / \mathrm{h}) \\
& \text { - } q_{p 1}^{f}=500(\mathrm{ped} / \mathrm{h})
\end{aligned}
$$

(a)

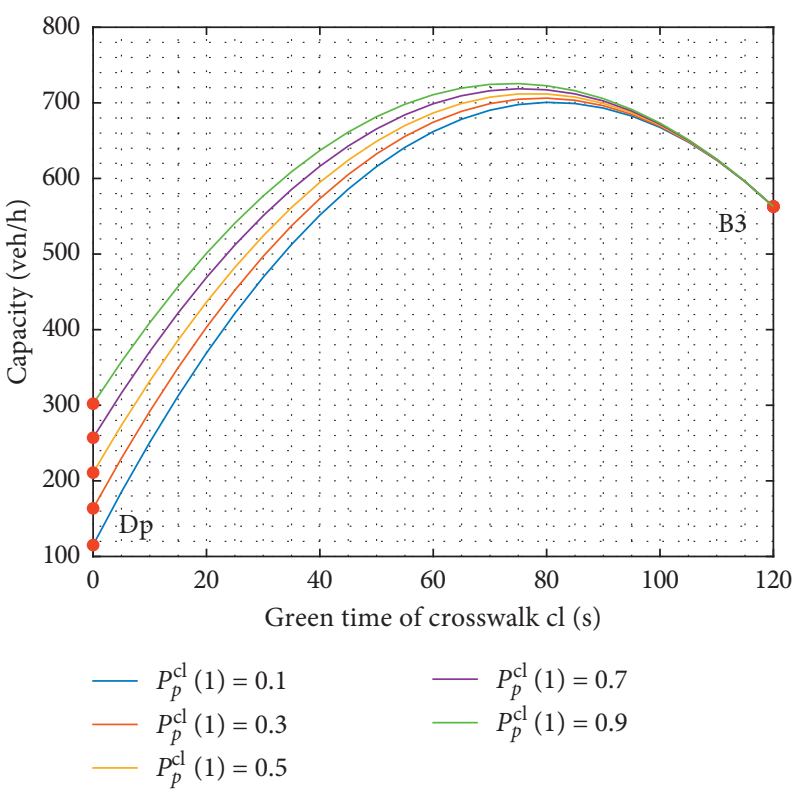

(c)

FIgURE 5: The sensitivity of capacity to green time radio. (d) $q_{p 2}^{f}=700($ ped $/ \mathrm{h})$.

two near crosswalks on one road. The schematic diagram of the pedestrian-vehicle interaction model of cellular automata is shown in Figure 6.

This paper adopts the same road geometric parameters and the size parameters of the traffic entity as Sun et al.'s [36] study on pedestrian-vehicle conflict based on cellular automata. Crosswalks are divided into square cell grids, each cell can only accommodate one person, and the cell width of the right-turn lane is equal to the side length of the cell of the crosswalk. Assume that the cellular side length of crosswalk is $l=0.4 \mathrm{~m}$ and the width of the exclusive right-turn lane is $2.8 \mathrm{~m}$, corresponding to the edge length of 7 cells of the crosswalk. The length of vehicle equals the summation of the side length of 15 cells $(6 \mathrm{~m})$.

(a) For the vehicle model, suppose that the maximum speed of a vehicle is $v_{\max }=40$; when the vehicle is less than $32 \mathrm{~m}$ ( 80 cells) away from the crosswalk, the maximum speed of the vehicle is $v_{\max }=20$. The maximum speed of vehicle on a crosswalk is $v_{\max }=10$. In this model, the open boundary condition is adopted. The vehicle enters the road with the arrival rate of $Q_{v}^{r-t}$. The update rule from $t$ to $t+1$ : acceleration: $v_{n+1} \longrightarrow \min \left(v_{n}+a, v_{\max }\right)$; deceleration: $\quad v_{n} \longrightarrow \min \left(v_{n}, d_{n}\right)$; randomization: with 


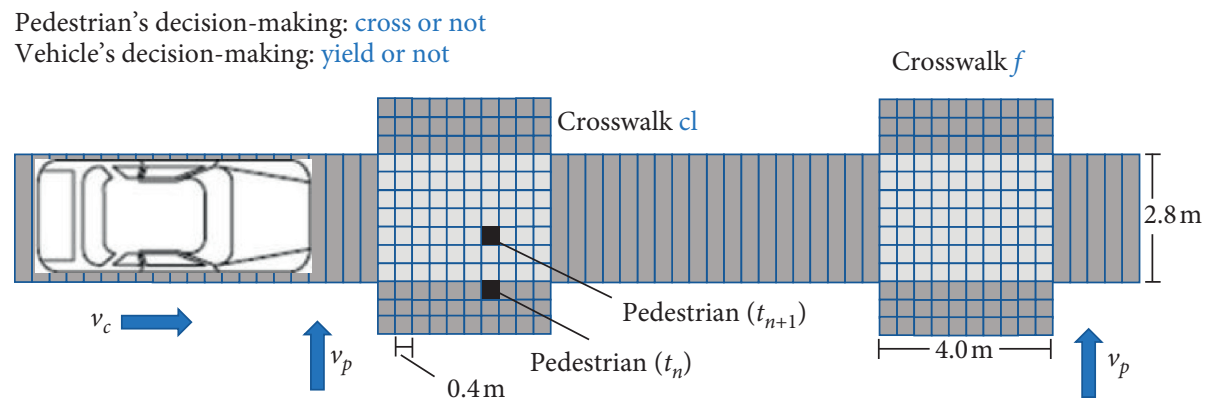

Figure 6: Schematic diagram of the cellular automata model for motor vehicles and pedestrians.

TABLE 7: Parameters in microscopic simulation.

\begin{tabular}{|c|c|c|c|c|c|c|}
\hline \multirow{2}{*}{$\begin{array}{l}\text { Parameter category } \\
\text { Traffic environment parameters }\end{array}$} & \multirow{2}{*}{\multicolumn{2}{|c|}{$\begin{array}{c}\text { Case } 1 \\
c(s) \\
120\end{array}$}} & \multirow{2}{*}{\multicolumn{2}{|c|}{$\begin{array}{c}\text { Case } 2 \\
t_{g}^{\mathrm{cl}}(\mathrm{s}) \\
27\end{array}$}} & \multicolumn{2}{|c|}{ Case 3} \\
\hline & & & & & $\begin{array}{c}t_{g}^{f}(\mathrm{~s}) \\
90\end{array}$ & $\begin{array}{c}L(\mathrm{~m}) \\
4.00\end{array}$ \\
\hline \multirow{4}{*}{ Arrival rates of pedestrians } & $q_{p 1}^{\mathrm{cl}}(\operatorname{ped} / \mathrm{h})$ & $0-1000$ & $q_{p 1}^{\mathrm{cl}}(\mathrm{ped} / \mathrm{h})$ & $0-1000$ & $q_{p 1}^{\mathrm{cl}}(\mathrm{ped} / \mathrm{h})$ & $0-1000$ \\
\hline & $q_{p 2}^{\mathrm{cl}}(\mathrm{ped} / \mathrm{h})$ & - & $q_{p 2}^{\mathrm{cl}}(\mathrm{ped} / \mathrm{h})$ & - & $q_{p 1}^{\mathrm{cl}}(\mathrm{ped} / \mathrm{h})$ & - \\
\hline & $q_{p 1}^{f}(\operatorname{ped} / \mathrm{h})$ & 200 & $q_{p 1}^{f}(\mathrm{ped} / \mathrm{h})$ & 200 & $q_{p 1}^{f}($ ped $/ \mathrm{h})$ & 200 \\
\hline & $q_{p 2}^{f}($ ped $/ \mathrm{h})$ & - & $q_{p 2}^{f}($ ped $/ \mathrm{h})$ & - & $q_{p 2}^{f}($ ped/h $)$ & - \\
\hline Probability of pedestrian's and vehicle's decision-making & $\begin{array}{l}P_{p}^{1}(1) \\
P_{v}^{1}(1) \\
P_{p}^{2}(1) \\
P_{v}^{2}(1)\end{array}$ & $\begin{array}{l}0.60 \\
0.40 \\
0.60 \\
0.40\end{array}$ & $\begin{array}{l}P_{p}^{1}(1) \\
P_{v}^{1}(1) \\
P_{p}^{2}(1) \\
P_{v}^{2}(1)\end{array}$ & $\begin{array}{l}0.70 \\
0.40 \\
0.60 \\
0.40\end{array}$ & $\begin{array}{l}P_{p}^{1}(1) \\
P_{v}^{1}(1) \\
P_{p}^{2}(1) \\
P_{v}^{2}(1)\end{array}$ & $\begin{array}{l}0.80 \\
0.40 \\
0.60 \\
0.40\end{array}$ \\
\hline
\end{tabular}

probability of $p$; and position update: $x_{n} \longrightarrow x_{n}+v_{n}$; (b) for the pedestrian model, pedestrians are divided into fast pedestrians and slow pedestrians with corresponding speed of 3 and 2, respectively. The pedestrian motion is divided into two steps: lane change and move forward. The two steps are processed through parallel rules and the speed of the pedestrian is recorded as $v_{i, j}$. The update rule from $t$ to $t+1$ : (1) lane change: the pedestrian chooses the path with the best condition by calculating $\max \left(d_{i, j}, d_{i, j}^{j-1}, d_{i, j}^{j+1}\right)$; (2) move forward: the walking speed $v_{i, j}=\min \left(v_{\max }^{p}, d_{i, j}\right)$; and (c) pedestrian-vehicle interaction rules: pedestrians and vehicles choose whether to pass or not according to the given probability in the model when they enter the conflict area.

For the evaluation of the proposed capacity model, this section constructed a case exclusive right-turn lane, which was microscopically simulated at $P_{p}^{1}(1)$ of $0.6,0.7$, and 0.8 , respectively. The case lane is located at a two-phase signalized intersection (see Figure 6). Parameters of the experimental lane in the microsimulation environment are as shown in Table 7.

The pedestrian arrival rate $q_{p 1}^{\text {cro }}$ changes from 0 ped/h to $1000 \mathrm{ped} / \mathrm{h}$ with $50 \mathrm{ped} / \mathrm{h}$ interval value. The simulation runs for $3600 \mathrm{~s}$ for each point, and after microsimulation, the capacity of the PV-RTC model was calculated. Under the condition that both $P_{p}^{1}(1)$ and $P_{p}^{2}(2)$ are 0.60 , the change of capacity with the change of the pedestrian arrival rate and the vehicle arrival rate is shown in Figure 7 . It can be seen from the figure that there is a critical value $Q_{v t}^{r-t}$, which divides the vehicle flow into two parts: free flow and congested flow. When $Q_{v}^{r-t}<Q_{\mathrm{vt}}^{r-t}$, the vehicle throughput increases linearly with the growth of $Q_{v}^{r-t}$; when $Q_{v}^{r-t}>Q_{\mathrm{vt}}^{r-t}$, the vehicle throughput reaches the saturation flow and remains unchanged; with the increase of $q_{\mathrm{p} 1}^{\mathrm{cl}}$, the saturation flow and critical value $Q_{\mathrm{vt}}^{r-t}$ of vehicles gradually decrease, but the decreasing range gradually decreases and finally reaches a stable value.

In addition, under the condition that both $q_{p 1}^{1}$ and $q_{p 2}^{1}$ are $200 \mathrm{ped} / \mathrm{h}$, the change of capacity with the change of yield rate and the vehicle arrival rate is shown in Figure 8. Similar to Figure 7, there is a critical value $Q_{v t}^{r-t}$, which divides the vehicle flow into two parts: free flow and congested flow. When $Q_{v}^{r-t}<Q_{v t}^{r-t}$, the vehicle throughput increases linearly with the growth of $Q_{v}^{r-t}$; when $Q_{v}^{r-t}>Q_{v t}^{r-t}$, it reaches saturation flow and remains unchanged; with the increase of $P_{v}^{\mathrm{cl}}(1)$, the vehicle saturation flow and critical value $Q_{v t}^{r-t}$ gradually decrease, and the decreasing range increases gradually.

The comparison between microscopic simulation and model estimation is shown in Figures 9(a)-9(c). At $P_{p}^{\mathrm{cl}}(1)$ of $0.6,0.7$, and 0.8 respectively, when the pedestrian arrival rate is the same, the error corresponding to the three figures tends to increase, and when the pedestrian arrival rate is large (close to $1000 \mathrm{ped} / \mathrm{h}$ ), the corresponding error reaches the maximum. A possible explanation for this phenomenon is that when the pedestrian arrival rate is relatively high, the number of pedestrians crossing the crosswalk will also increase, which means that the number and probability of 


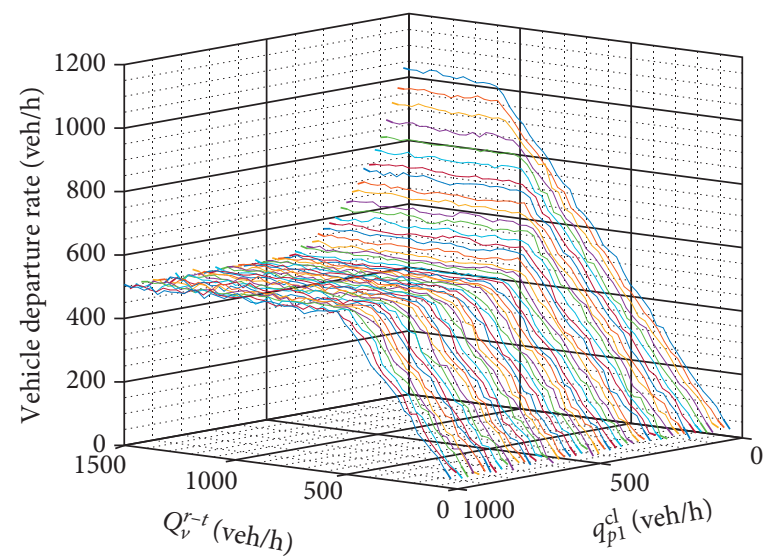

Figure 7: Relationship among $C_{r}, q_{p 1}^{\mathrm{cl}}$, and $Q_{v}^{r-t}$.

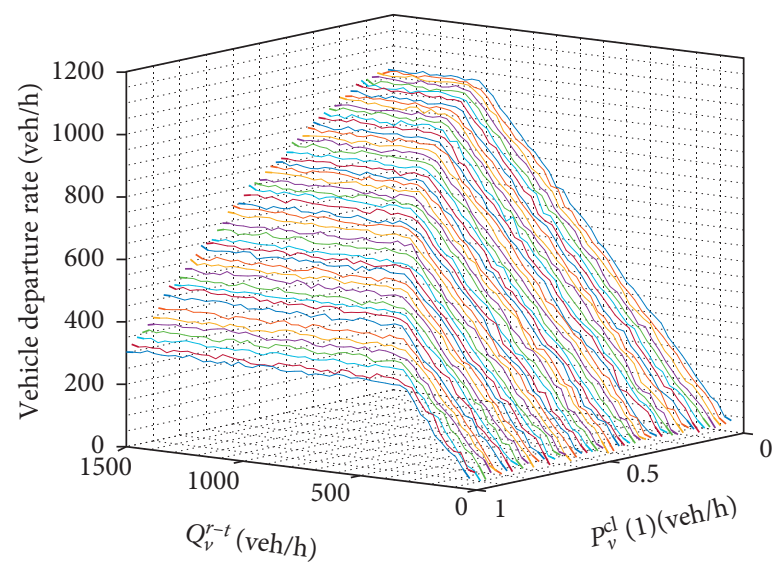

Figure 8: Relationship among $C_{r}, P_{v}^{\mathrm{cl}}(1)$, and $Q_{v}^{r-t}$.

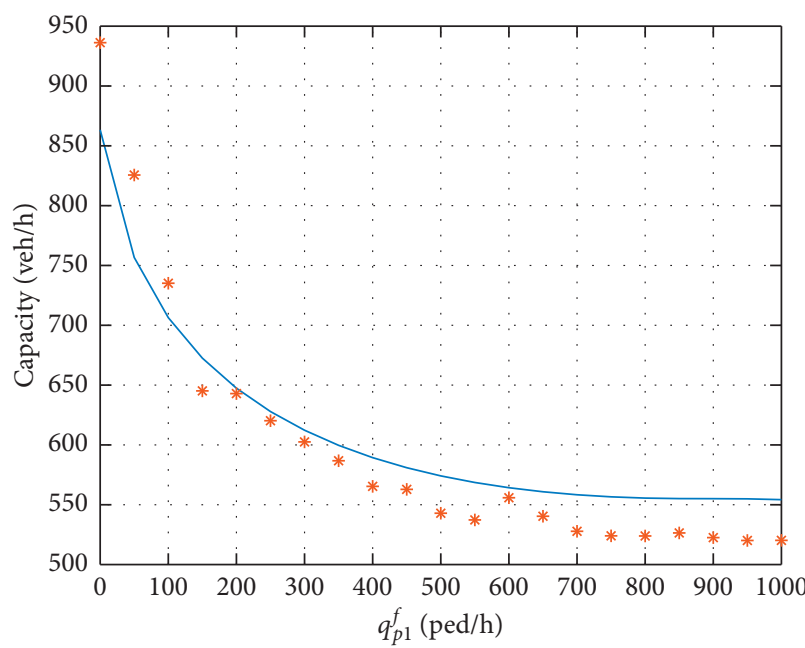

- Model estimation

* CA simulation

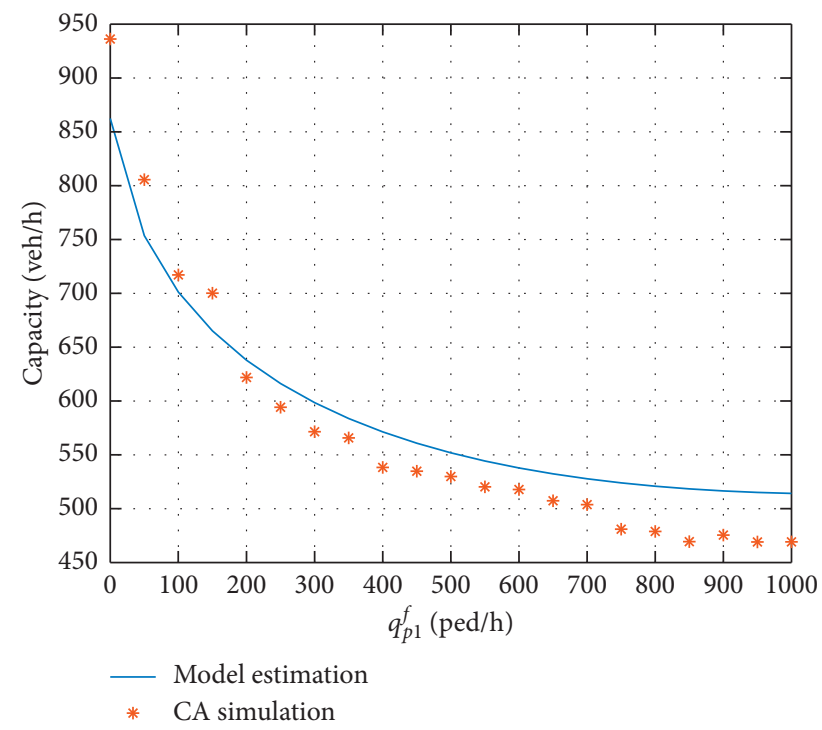

(b)

Figure 9: Continued. 


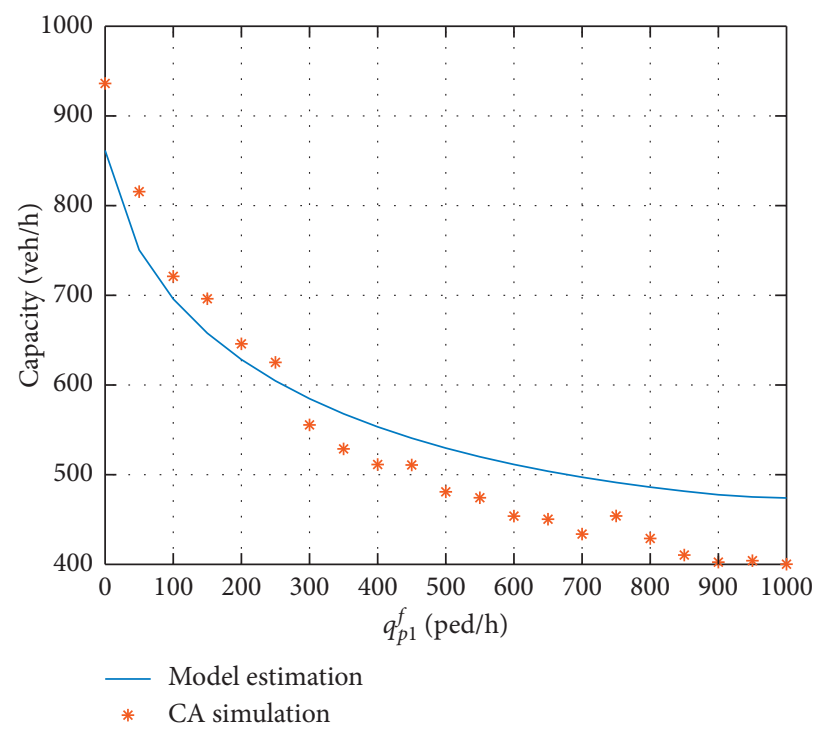

(c)

FIGURE 9: A comparison between microscopic simulation and model estimation. (a) $P_{p}^{1}(1)=0.6$. (b) $P_{p}^{1}(1)=0.7$. (c) $P_{p}^{1}(1)=0.8$.

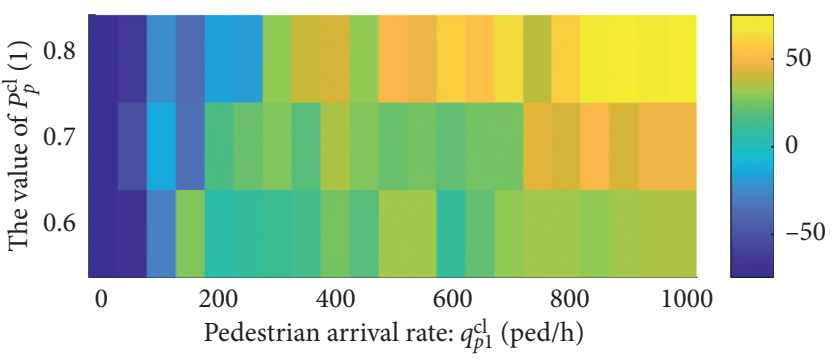

(a)

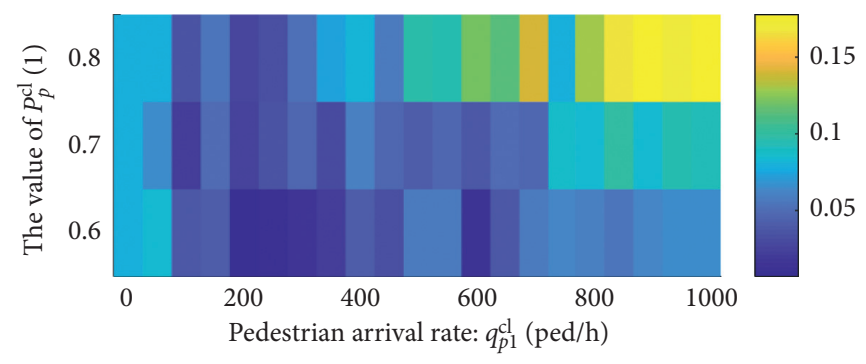

(b)

Figure 10: (a) Absolute error and (b) relative error between microscopic simulation and model estimation.

pedestrians choosing to cross at the end of the pedestrian green time in each cycle will also increase overall. In addition, when the pedestrian arrival rate is not very high, the trend of capacity change of the two models is basically the same.

Figure 10 provides the deviation between microsimulation calculation and model estimation. It can be seen that the absolute error of the capacity is less than $70 \mathrm{veh} / \mathrm{h}$ overall, and the relative error is less than $15 \%$ except when $P_{p}^{\mathrm{cl}}(1)$ is 0.8 and $q_{p 1}^{\mathrm{cl}}$ is close to $1000 \mathrm{ped} / \mathrm{h}$. A comparison of the two results reveals it is reasonable to evaluate the exclusive right-turn lane capacity at signalized intersections using the PV-RTC model constructed.

\section{Conclusions and Future Research}

This paper proposes an exclusive right-turn lane capacity model considering pedestrian-vehicle interaction (PVRTC). Firstly, a pedestrian-vehicle interaction model at crosswalk is proposed. Then, according to situations (3) and (4) during pedestrian-vehicle interaction process, a pedestrian-vehicle interaction evolutionary model based on game theory is established. Finally, based on the probabilities of 6 kinds of pedestrian-vehicle interaction situations and the basic idea of the stop line method, the PV-RTC model is established. After that, a pedestrian-vehicle interaction model of cellular automata for exclusive right-turn lane is established and its simulation results are compared with the results of the PV-RTC model. The results show that the relative error between the cellular automata model and PVRTC model is less than $15 \%$ overall, which verifies the validity of the PV-RTC model.

The PV-RTC model proposed in this paper has the following limitations: (a) in the model, pedestrians are divided into three categories (the young, the middle-aged, and the old). However, pedestrians have many other characteristic differences (e.g., gender and behavior) in reality. More detailed division of pedestrians means that the utility function in the pedestrian-vehicle interaction model is more consistent with the actual situation; (b) in order to focus on the influence of the pedestrian-vehicle interaction process on road capacity, the influence of nonmotor vehicle is not considered in the PV-RTC model. In future studies, their interaction with traffic flow should be comprehensively considered.

The change of the interaction mechanism between pedestrians and vehicles will have a systematic impact on traffic 
operation. Future research can be extended to the following three aspects.

(a) Research on the theory of pedestrian-vehicle interaction: pedestrian-vehicle interaction situations occur in specific traffic environment. When the traffic environment (e.g., road conditions and traffic regulations) changes, the interaction process between pedestrians and vehicles will change accordingly. How to establish a more practical theory of pedestrian-vehicle interaction is a worthy research direction.

(b) This study only establishes the exclusive right-turn lane capacity model based on the pedestrian-vehicle interaction process. The influence of pedestrians on traffic flow at intersections exists in almost every lane, and a more accurate intersection capacity is an urgent need for intelligent transportation systems and refined traffic control. Therefore, the research based on pedestrian-vehicle interaction can be extended to the whole intersection and other traffic problems (e.g., intersection signal timing, level of service (LOS), and vehicle delay).

(c) In the sensitivity analysis section of this paper, some qualitative analysis has been conducted on the relationship between the yielding rate, efficiency, and safety, and it can be found that there is some internal relationship between traffic environment and the safety and efficiency during the pedestrian-vehicle interaction process. In this aspect, the quantitative relationship between them can be further studied in the future, so as to provide policy suggestions for the coordination of policy formulation and facility improvement for traffic management departments.

\section{Data Availability}

All the data used to support the findings of this study are included within the article.

\section{Conflicts of Interest}

The authors declare that they have no conflicts of interest.

\section{Acknowledgments}

This work was financially supported by the Shanghai Sailing Program (Grant no. 19YF1435100).

\section{References}

[1] A. N. Arshi, W. K. M. Alhajyaseen, H. Nakamura, and $\mathrm{X}$. Zhang, "A comparative study on the operational performance of four-leg intersections by control type," Transportation Research Part A: Policy and Practice, vol. 118, pp. 52-67, 2018.

[2] Z. W. Zheng, K. Li, J. Q. Xu et al., Advanced Urban Transport, China Communications Press, Beijing, China, 1998.

[3] Y. P. Zhang, Highway Capacity Theory, Harbin Institute of Technology Press, Harbin, China, 2007.
[4] J. Y. Xu, Design of Urban Road Engineering, China Water \& Power Press, Beijing, China, 2005.

[5] X. U. Ji-qian and C. Xue-wu, Fundamentals of Traffic Engineering, China Communications Press, Beijing, China, 3rd edition, 2008.

[6] CJJ 37-90, Design Regulation of Urban Road Engineering, Beijing, China, 2016.

[7] T. R. Board, Highway Capacity Manual, Transportation Research Board, Washington, DC, USA, 2010.

[8] S. Teply, D. I. Allingham, D. B. Richardson et al., Canadian Capacity Guide for Signalized Intersections, Institute of Transportation Engineers, Ottawa, Canada, 3rd edition, 2008.

[9] R. T. Luttinen and R. Nevala, Capacity and Level of Service of Finnish Signalized Intersections, Finnish Road Administration, Helsinki, Finland, 2002.

[10] Japan Road Association, Highway Capacity, Japan Road Association, Tokyo, Japan, 1984.

[11] Federal Highway Research Institute, HBS 2010: German Highway Capacity Manual, FGSV Verlag GmbH, Koeln, Germany, 2010.

[12] X. G. Yang, J. Zhao, and W. J. Ma, "Review on calculation method for signalized intersection capacity," China Journal of Highway and Transport, vol. 27, no. 5, pp. 148-157, 2014.

[13] K. M. Kockelman and R. A. Shabih, "Effect of light-duty trucks on the capacity of signalized intersections," Journal of Transportation Engineering, vol. 126, no. 6, pp. 506-512, 2000.

[14] J. A. Sosin, "Delays at intersections controlled by fixed-cycle traffic signals," Traffic Engineering and Control, vol. 21, no. 8/ 9, pp. 407-413, 1980.

[15] B. P. Ingrid, F. R. John, M. B. Karin et al., "Relationship of lane width to saturation flow rate on urban and suburban signalized intersection approaches," Transportation Research Record, vol. 2027, no. 1, pp. 45-51, 2007.

[16] D. Wu, J. Zhao, and X. G. Yang, "Impact of bus stop on signalized intersection capacity," in Proceedings of the Second International Conference on Intelligent Computation Technology and Automation, IEEE Computer Society, Changsha, China, pp. 619-624, 2009.

[17] D. Torbic and L. Elefteriadou, "Effects of driver population on the traffic operational performance of signalized intersections," in Proceedings of the Fourth International Symposium on Highway Capacity, Transportation Research Board, Washington, DC, USA, pp. 336-347, 2000.

[18] M. S. Tarawneh and T. M. Tarawneh, "Effect on utilization of auxiliary through lanes of downstream right-turn volume," Journal of Transportation Engineering, vol. 128, no. 5, pp. 458-464, 2002.

[19] Z. Z. Tian and N. Wu, "Probabilistic model for signalized intersection capacity with a short right-turn lane," Journal of Transportation Engineering, vol. 132, no. 3, pp. 205-212, 2006.

[20] X. G. Yang, J. Zhao, and X. F. Yu, "Calculation methods of section capacity with consideration of effect of access traffic," China Journal of Highway and Transport, vol. 22, no. 5, pp. 83-88, 2009.

[21] X. G. Yang, J. Zhao, and X. F. Yu, "Impact of upstream weaving segment on signalized intersection capacity," in Proceedings of the 2008 International Conference on Intelligent Computation Technology and Automation (ICICTA), Hunan, China, 2008.

[22] X. M. Chen, C. F. Shao, and Y. Hao, "Influence of pedestrian traffic on capacity of right-turning movements at signalized intersections," Transportation Research Record, vol. 2073, pp. 59-67, 2008. 
[23] J. E. Coeymans and J. C. Herrera, "Estimating values for traffic parameters in turning lanes," Transportation Research Record, vol. 1852, pp. 47-54, 2003.

[24] M. Nekoui, H. Pishro-nik, and D. Ni, "The effect of intellidrive on the efficiency of highway transportation systems," International Journal of Vehicular Technology, vol. 2011, Article ID 653542, 11 pages, 2011.

[25] P. D. Prevedouros and K. Chang, "Potential effects of wet conditions on signalized intersection LOS," Journal of Transportation Engineering, vol. 131, no. 12, pp. 898-903, 2005.

[26] X. M. Chen, C. F. Shao, and Z. H. Xiong, "Reliability of ssignalized intersection capacity under mixed traffic," China Journal of Highway and Transport, vol. 21, no. 4, pp. 99-104, 2008.

[27] J. Mcmahon, T. Goddard, and A. Adkins, "Racial bias in driver yielding behavior at crosswalks," Transportation Research Part F: Traffic Psychology and Behaviour, vol. 33, pp. 1-6, 2015.

[28] E. A. Bourquin, R. W. Emerson, D. Sauerburger, and J. M. Barlow, "Conditions that influence drivers' behaviors at roundabouts: increasing yielding for pedestrians who are visually impaired," Journal of Visual Impairment \& Blindness, vol. 112, no. 1, pp. 61-71, 2018.

[29] Y. Zheng and L. Elefteriadou, "A model of pedestrian delay at unsignalized intersections in urban networks," Transportation Research Part B: Methodological, vol. 100, pp. 138-155, 2017.

[30] S. H. Dou and J. Q. Gou, "A study on the behavioral evolution of pedestrian and auto-drivers in the signalized intersection," Journal of Beijing Jiaotong University (Social Sciences Edition), vol. 14, no. 4, pp. 66-72, 2015.

[31] S. Stapleton, T. Kirsch, and T. J. Gates, "Factors affecting driver yielding compliance at uncontrolled midblock crosswalks on low-speed roadways," Transportation Research Record: Journal of the Transportation Research Board, vol. 2661, no. 1, pp. 95-102, 2018.

[32] M. Sucha, D. Dostal, and R. Risser, "Pedestrian-driver communication and decision strategies at marked crossings," Accident Analysis \& Prevention, vol. 102, pp. 41-50, 2017.

[33] F. Kay, B. Marcus, A. Raul et al., "Will you stop for me? An exploration of characteristics associated with a driver's decision to stop for a pedestrian in a crosswalk with a rectangular rapid-flashing beacon," TRB Meeting, vol. 87, no. 3, pp. 36-41, 2017.

[34] R. V. Houten, J. E. L. Malenfant, and B. Huitema, "Highvisibility enforcement on driver compliance with pedestrian right-of-way laws: 4-year follow-up," Transportation Research Record: Journal of the Transportation Research Board, vol. 2660, no. 1, pp. 58-65, 2018.

[35] L. Y. Wei, Y. F. Cui, and D. Y. Li, "Evolution mechanism of conflict between pedestrian and vehicle based on evolutionary game theory," Acta Physica Sinica, vol. 67, no. 19, pp. 37-49, 2018.

[36] B. Jia, Z. Sun, and X. G. Li, "The study of the interference between pedestrians and vehicles based on celluar automation model," Acta Physica Sinica, vol. 61, no. 10, Article ID 100508, 2012. 